\title{
High-Throughput Study of Lattice Thermal Conductivity in Binary Rocksalt and Zinc Blende Compounds Including Higher-Order Anharmonicity
}

\author{
Yi Xia®, ${ }^{1, *}$ Vinay I. Hegde, ${ }^{1}$ Koushik Palø, ${ }^{1}$ Xia Hua, ${ }^{1}$ Dale Gaines $\odot,{ }^{1}$ Shane Patelø, ${ }^{1}$ \\ Jiangang He, ${ }^{1}$ Muratahan Aykol@ ${ }^{2}$ and Chris Wolverton ${ }^{1, \dagger}$ \\ ${ }^{1}$ Department of Materials Science and Engineering, Northwestern University, \\ Evanston, Illinois 60208, USA \\ ${ }^{2}$ Toyota Research Institute, Los Altos, California 94022, USA
}

(Received 26 May 2020; revised 21 August 2020; accepted 21 September 2020; published 10 November 2020)

\begin{abstract}
Thermal transport phenomena are ubiquitous and play a critical role in the performance of various microelectronic and energy-conversion devices. Binary rocksalt and zinc blende compounds, despite their rather simple crystal structures, exhibit an extraordinary range of lattice thermal conductivity $\left(\kappa_{\mathrm{L}}\right)$ spanning over 3 orders of magnitude. A comprehensive understanding of the underlying heat transfer mechanism through the development of microscopic theories is therefore of fundamental importance, yet it remains elusive because of the challenges arising from explicitly treating higher-order anharmonicity. Recent theoretical and experimental advances have revealed the essential role of quartic anharmonicity in suppressing heat transfer in zinc blende boron arsenide (BAs) with ultrahigh $\kappa_{\mathrm{L}}$. However, critical questions concerning the general effects of higher-order anharmonicity in the broad classes and chemistries of binary solids are still unanswered. Using our recently developed high-throughput phonon framework based on first-principles density functional theory calculations, we systematically investigate the lattice dynamics and thermal transport properties of 37 binary compounds with rocksalt and zinc blende structures at room temperature, with a particular focus on unraveling the impacts of quartic anharmonicity on $\kappa_{\mathrm{L}}$. Our advanced theoretical model for computing $\kappa_{\mathrm{L}}$ embraces current state-of-the-art methods, featuring a complete treatment of quartic anharmonicity for both phonon frequencies and lifetimes at finite temperatures, as well as contributions from off-diagonal terms in the heat-flux operator. We find the impacts of quartic anharmonicity on $\kappa_{\mathrm{L}}$ to be strikingly different in rocksalt and zinc blende compounds, owing to the countervailing effects on finitetemperature-induced shifts in phonon frequencies and scattering rates. By correlating $\kappa_{\mathrm{L}}$ with the phonon scattering phase space, we outline a qualitative but efficient route to assess the importance of four-phonon scattering from harmonic phonon calculations. Among notable examples, in zinc blende $\mathrm{HgTe}$, we identify an unprecedented sixfold reduction in $\kappa_{\mathrm{L}}$ due to four-phonon scattering, which dominates over the three-phonon scattering in the acoustic region at room temperature. We also demonstrate a possible breakdown of the phonon gas model in rocksalt $\mathrm{AgCl}$, wherein the phonon states are significantly broadened due to strong intrinsic anharmonicity, inducing off-diagonal contributions to $\kappa_{\mathrm{L}}$ comparable to the diagonal ones. The deep physical insights gained in this work can be used to guide the rational design of thermal management materials.
\end{abstract}

DOI: $10.1103 /$ PhysRevX.10.041029

Subject Areas: Energy Research, Materials Science, Semiconductor Physics

\section{INTRODUCTION}

Thermal conductivity, heat conduction under a finitetemperature gradient, is of vital importance in various modern technologies, including transistors, photovoltaics,

\footnotetext{
*yimaverickxia@gmail.com

†c-wolverton@northwestern.edu
}

Published by the American Physical Society under the terms of the Creative Commons Attribution 4.0 International license. Further distribution of this work must maintain attribution to the author(s) and the published article's title, journal citation, and DOI. and thermoelectric devices [1,2]. In particular, increasing power density in microprocessors demands efficient thermal management, where high thermal conductivity is desired to remove heat away from the "hot spots" [3]. On the other hand, irreversible heat transfer needs to be minimized in order to achieve optimal energy conversion efficiency in the application of thermoelectric materials [4]. Therefore, semiconducting materials of particular interest are those exhibiting extreme thermal conductivities, with either very high or very low lattice thermal conductivities $\left(\kappa_{\mathrm{L}}\right)$. Surprisingly, these rather extreme material properties have been discovered in simple binary cubic compounds with rocksalt and zinc blende structures (hereinafter 
referred to as binary rocksalts and zinc blendes), which have $\kappa_{\mathrm{L}}$ spanning more than 3 orders of magnitude [3]. Prominent examples include lead chalcogenides $(\mathrm{PbS} / \mathrm{Se} / \mathrm{Te})$, which are known for their superior thermoelectric properties with very low $\kappa_{\mathrm{L}}$ [5], and boron arsenide (BAs) with ultrahigh $\kappa_{\mathrm{L}}$, second only to that of diamond among bulk compounds comprised of naturally occurring elements [6-8].

Despite the structural simplicity of binary rocksalts and zinc blendes, comprehensive experimental characterization of the thermal transport properties and theoretical understanding of the lattice dynamics in these compounds are still quite challenging. Theoretically, extensive lattice dynamics theories were formulated decades ago [9-13]. Their application in modeling $\kappa_{\mathrm{L}}$ of real materials in a firstprinciples manner has only been brought to life recently, thanks to the development of density functional theory (DFT) $[14,15]$ and high-performance computing. Widely used modeling approaches are based either on molecular dynamics (MD) or anharmonic lattice dynamics (ALD). MD-based methods are, in principle, more general because of the inclusion of all anharmonic terms and the existence of a universal expression for $\kappa_{\mathrm{L}}$ in the Green-Kubo framework [16,17]. However, their applications in modeling $\kappa_{\mathrm{L}}$ for a large number of compounds are still impeded by the lack of computationally efficient and accurate force fields, the latter of which are usually specific to a given system, not to mention the challenge arising from fully incorporating the quantum effects [18-20]. These challenges can be partially overcome by an alternative theoretical framework that combines ALD and the PeierlsBoltzmann transport equation (PBTE) [21-27], wherein phonon states carrying heat are treated as quasiparticles with well-defined energies and finite lifetimes, in the spirit of the phonon gas model (PGM) [28]. Recently, the ALDPBTE approach has been widely adopted to model and analyze $\kappa_{\mathrm{L}}$ in a variety of crystalline compounds, successfully unraveling the microscopic mechanism underlying both very high $\kappa_{\mathrm{L}}$ [29-31] and very low $\kappa_{\mathrm{L}}$ [32-40].

The application of the ALD-PBTE approach has led to the prediction of an anomalously large $\kappa_{\mathrm{L}}$ in zinc blende BAs with a value higher than diamond above room temperature. The microscopic origin has been attributed to the weak three-phonon (3ph) interactions [30]. However, a significantly lower $\kappa_{\mathrm{L}}$ (but still high) was reported by experiments [41,42], which was initially attributed to the presence of extrinsic phonon scattering from defects and grain boundaries [43]. Recent theoretical advances in ALD calculations have demonstrated that higher-than-third-order anharmonicity, or specifically, four-phonon (4ph) scattering, is responsible for a roughly $40 \%$ reduction in the $\kappa_{\mathrm{L}}$ of BAs compared to that predicted considering only $3 \mathrm{ph}$ scattering [44]. Nevertheless, $\kappa_{\mathrm{L}}$ of naturally occurring BAs still reaches over $1000 \mathrm{~W} /(\mathrm{m} \cdot \mathrm{K})$ [44] at room temperature. This improved theoretical prediction was subsequently confirmed by a combination of (i) advanced experimental synthesis of high-quality single-crystal BAs samples and (ii) thermal conductivity measurements based on time-domain thermoreflectance or frequency-domain thermoreflectance [6-8]. In addition, most recent firstprinciples studies have revealed the importance of thermal expansion, anharmonic phonon renormalization (APRN), $4 \mathrm{ph}$ scattering, and the interplay between them to accurately estimate $\kappa_{\mathrm{L}}$. Explicit examples have been demonstrated by the nontrivial temperature dependence of $\kappa_{\mathrm{L}}$ in strongly anharmonic PbTe [45] and the anomalously weak temperature dependence of phonon frequency in $\mathrm{NaCl}$ [46]. In fact, it has been found that in some cases, the agreement between $\kappa_{\mathrm{L}}$ obtained from calculations considering only 3 ph scattering and experiments is likely due to cancellation of errors [45].

Given the complex interplay of various factors contributing to $\kappa_{\mathrm{L}}$, despite years of research into simple binary rocksalts and zinc blendes, there are still a plethora of unexplored avenues and critical unanswered questions regarding their transport properties. One of the outstanding challenges is to achieve a comprehensive understanding of the effects of higher-order (e.g., quartic) anharmonicity on lattice heat transport. It is worth mentioning that the theoretical formalism for explicitly treating higher-order anharmonicity was developed about a half century ago $[10,47-50]$. However, due to formidable computational cost and theoretical complexity, quartic anharmonicity has rarely been incorporated in first-principles calculations of $\kappa_{\mathrm{L}}$ within the framework of ALD and PBTE, and it is limited to only a few case-by-case studies [44-46,51-59]. Specifically, the following fundamental questions remain unexplored:

(i) How does quartic anharmonicity generally affect $\kappa_{\mathrm{L}}$, and does the inclusion of quartic anharmonicity always reduce $\kappa_{\mathrm{L}}$ ?

(ii) When is quartic anharmonicity important? Is there a way to assess the significance of $4 \mathrm{ph}$ scattering using only harmonic calculations?

(iii) Will quartic anharmonicity be critical enough to cause a breakdown of the phonon gas model and modify the microscopic thermal transport mechanism?

Question (i) arises from the complex role of $4 \mathrm{ph}$ interactions in that they not only lead to additional phonon scattering rates but also induce shifts in phonon frequencies at finite temperature; the net change in $\kappa_{\mathrm{L}}$ caused by $4 \mathrm{ph}$ interactions is thus nontrivial. (For conceptual clarity, we adopt a convention in this study that " $4 \mathrm{ph}$ interactions" refer to both frequency shifts and scattering rates, while " $4 \mathrm{ph}$ scattering" refers to scattering rates only.) Question (ii) could be answered by a practical approach to assess the effects of $4 \mathrm{ph}$ interactions efficiently, presumably from relatively cheaper harmonic calculations. Question (iii) addresses the validity of the phonon quasiparticle picture adopted in PGM and other 
TABLE I. Calculated lattice thermal conductivities in units of $\mathrm{W} /(\mathrm{m} \cdot \mathrm{K})$ using various levels of theory, namely, $\kappa_{3 \mathrm{ph}}^{\mathrm{HA}}, \kappa_{3 \mathrm{ph}}^{\mathrm{SCPH}}$, and $\kappa_{3,4 \mathrm{ph}}^{\mathrm{SCPH}}$, for selected binary compounds in rocksalt and zinc blende structures compared with experimental values. All theoretical values are calculated at $300 \mathrm{~K}$ using the PBE functional, except those values in parentheses for $\mathrm{PbTe}, \mathrm{AgCl}$, and $\mathrm{HgTe}$, which are calculated using the PBEsol functional. Thermal expansion is also included (using experimental lattice parameters at $300 \mathrm{~K}$ ) in the case of LiH, $\mathrm{PbTe}$, and $\mathrm{AgCl}$, as they display much larger thermal expansion than that of $\mathrm{HgTe}$. The absence of $\kappa_{3 \mathrm{ph}}^{\mathrm{HA}}$ for $\mathrm{AgCl}$ is due to the presence of imaginary frequencies in harmonic phonons. Note that the ground-state structures of $\mathrm{HgS}$ and $\mathrm{CdSe}$ are not zinc blende, and hence no experimental $\kappa_{\mathrm{L}}$ is available. We include hypothetical zinc blende $\mathrm{HgS}$ and $\mathrm{CdSe}$ to compare the trend across $\mathrm{S} / \mathrm{Se} / \mathrm{Te}$ compounds.

\begin{tabular}{|c|c|c|c|c|c|c|c|c|c|c|c|c|c|c|c|c|c|c|c|}
\hline Rocksalts & $\mathrm{MgO}$ & $\mathrm{CaO}$ & $\mathrm{SrO}$ & $\mathrm{BaO}$ & $\mathrm{LiH}$ & $\mathrm{LiF}$ & $\mathrm{NaF}$ & $\mathrm{NaCl}$ & $\mathrm{NaBr}$ & $\mathrm{NaI}$ & $\mathrm{KF}$ & $\mathrm{KCl}$ & $\mathrm{KBr}$ & KI & $\mathrm{RbCl}$ & $1 \mathrm{PbS}$ & $\mathrm{PbSe}$ & $\mathrm{PbTe}$ & $\mathrm{AgCl}$ \\
\hline$\kappa_{3 \mathrm{ph}}^{\mathrm{HA}}$ & 52.1 & 21.3 & 9.0 & 2.8 & $22.9(15.6)$ & 11.3 & 20.6 & 7.2 & 2.9 & 1.7 & 5.9 & 6.6 & 2.2 & 1.3 & 1.7 & 1.3 & 1.1 & $1.6(2.1)$ & (N/A) \\
\hline$\kappa_{3 \mathrm{ph}}^{\mathrm{SCPH}}$ & 58.7 & 25.1 & 11.0 & 4.4 & $33.1(24.5)$ & 15.4 & 26.4 & 9.7 & 3.6 & 2.5 & 7.1 & 8.9 & 3.0 & 2.0 & 2.2 & 2.2 & 2.0 & $2.7(3.4)$ & $(0.55)$ \\
\hline$\kappa_{3,4 \mathrm{ph}}^{\mathrm{SCPH}}$ & 50.1 & 22.2 & 9.9 & 3.3 & $25.8(18.3)$ & 11.4 & 14.9 & 5.4 & 2.6 & 1.6 & 5.0 & 4.5 & 2.0 & 1.4 & 1.7 & 1.5 & 1.5 & $1.7(2.3)$ & $(0.41)$ \\
\hline $\begin{array}{l}\kappa_{\mathrm{L}}^{\exp } \\
\text { Reference }\end{array}$ & $\begin{array}{l}52 \\
{[66]}\end{array}$ & $\begin{array}{c}30 \\
{[66]}\end{array}$ & $\begin{array}{l}10 \\
{[66]}\end{array}$ & $\begin{array}{c}3 \\
{[66]}\end{array}$ & $\begin{array}{l}14.7 \\
{[67]}\end{array}$ & $\begin{array}{l}16.1 \\
{[68]}\end{array}$ & $\begin{array}{l}18.5 \\
{[69]}\end{array}$ & $\begin{array}{c}7.2 \\
{[70]}\end{array}$ & $\begin{array}{l}2.9 \\
{[71]}\end{array}$ & $\begin{array}{c}1.9 \\
{[70]}\end{array}$ & $\begin{array}{l}7.8 \\
{[72]}\end{array}$ & $\begin{array}{c}7.2 \\
{[73]}\end{array}$ & $\begin{array}{l}3.4 \\
{[73]}\end{array}$ & $\begin{array}{c}2.8 \\
{[73]}\end{array}$ & $\begin{array}{l}2.9 \\
{[73]}\end{array}$ & $\begin{array}{c}2.5 \\
{[74]}\end{array}$ & $\begin{array}{l}1.6 \\
{[74]}\end{array}$ & $\begin{array}{c}2.2 \\
{[75]}\end{array}$ & $\begin{array}{l}1.1 \\
{[76]}\end{array}$ \\
\hline Zinc blendes & AlAs & $\mathrm{AlSb}$ & $\mathrm{BN}$ & BP & $\mathrm{BAs}$ & $\mathrm{CdSe}$ & $\mathrm{CdTe}$ & $\mathrm{GaP}$ & $\mathrm{GaSb}$ & InP & InAs & $\mathrm{InSb}$ & $\mathrm{ZnS}$ & $\mathrm{ZnSe}$ & $\mathrm{ZnTe}$ & $\mathrm{HgS}$ & $\mathrm{HgSe}$ & $\mathrm{HgTe}$ & \\
\hline$\kappa_{3 \mathrm{ph}}^{\mathrm{HA}}$ & 93.1 & 88.8 & 776.7 & 415.6 & 2155.6 & 13.5 & 7.6 & 118.8 & 33.2 & 90.9 & 28.5 & 16.8 & 42.1 & 19.2 & 21.4 & 4.5 & 4.6 & $8.1(12.8)$ & \\
\hline$\kappa_{3 \mathrm{ph}}^{\mathrm{SCPH}}$ & 88.9 & 84.3 & 774.0 & 413.8 & 1997.4 & 13.1 & 7.5 & 117.3 & 31.8 & 87.3 & 27.3 & 15.7 & 41.5 & 18.8 & 21.3 & 5.3 & 5.6 & $8.7(14.2)$ & \\
\hline$\kappa_{3,4 \mathrm{ph}}^{\mathrm{SCPH}}$ & 67.2 & 41.2 & 764.2 & 397.7 & 1093.9 & 7.4 & 5.0 & 84.1 & 22.5 & 51.0 & 19.4 & 11.1 & 29,6 & 14.9 & 15.7 & 1.8 & 1.7 & $1.8(2.3)$ & \\
\hline$\kappa_{\mathrm{L}}^{\exp }$ & 91 & $46 / 56$ & 740 & $400 / 460$ & $1300 / 1000$ & N/A & 7.5 & $76 / 100$ & 37.8 & 67 & 27.3 & 16.6 & 27 & 19 & 18 & N/A & 1.7 & $1.9 / 2.6$ & \\
\hline Reference & [77] & {$[78] /[79]$} & {$[80]$} & {$[81] /[82]$} & {$[6] /[7]$} & N/A & [83] & {$[79] /[67]$} & {$[78]$} & [78] & [78] & {$[78]$} & [83] & {$[83]$} & [83] & N/A & [83] & {$[83] /[84]$} & \\
\hline
\end{tabular}

possible thermal transport mechanisms beyond PGM, including the off-diagonal terms from a more general expression of the heat-flux operator.

To shed light on these questions, we systematically investigate the impacts of quartic anharmonicity on the lattice dynamics and thermal transport properties of 19 binary rocksalts and 18 binary zinc blendes (see Table I) by means of our recently developed high-throughput framework for modeling material properties at finite temperatures [60]. This first-principles-based framework embraces stateof-the-art theoretical models and techniques, featuring (i) efficient and accurate construction of high-order anharmonic interatomic force constants (IFCs) using compressive sensing lattice dynamics (CSLD) [61-63], (ii) advanced anharmonic lattice dynamics with full treatment of quartic anharmonicity, accounting for both phonon frequency shifts and scattering rates [51,52], and (iii) a unified lattice thermal transport model including both diagonal and off-diagonal contributions to $\kappa_{\mathrm{L}}$ from the heat-flux operator $[64,65]$.

We study the impacts of quartic anharmonicity on $\kappa_{\mathrm{L}}$ in 37 selected binary rocksalts and zinc blendes at room temperature, along a ladder [see Fig. 2(a)] of approximations for modeling $\kappa_{\mathrm{L}}$, as described in Sec. II A. Our work shows that the impact of quartic anharmonicity in rocksalts differs significantly, both qualitatively and quantitatively, from that in zinc blendes: Quartic anharmonicity on top of the cubic anharmonicity always reduces $\kappa_{\mathrm{L}}$ in binary zinc blendes to varying degrees, while it results in either reduced or increased $\kappa_{\mathrm{L}}$ in binary rocksalts. We find that this surprising finding can systematically and significantly reduce discrepancies between theoretically predicted and experimentally measured $\kappa_{\mathrm{L}}$. Furthermore, we demonstrate a clear correlation between the relative change in $\kappa_{\mathrm{L}}$ and $3 \mathrm{ph} / 4 \mathrm{ph}$ scattering phase space, which can be obtained from cheap harmonic calculations. We also discover unprecedentedly strong $4 \mathrm{ph}$ scattering in zinc blende $\mathrm{HgSe} / \mathrm{Te}$, whose $4 \mathrm{ph}$ scattering rates unexpectedly dominate over $3 \mathrm{ph}$ scattering rates in the acoustic region. Contrary to a general consensus that PGM is valid in simple and ordered compounds at low temperatures, we show, in a case study of rocksalt $\mathrm{AgCl}$, the possible breakdown of $\mathrm{PGM}$ at room temperature, even in the context of anharmonically renormalized phonons. The strong phonon broadening due to intrinsic anharmonicity gives rise to off-diagonal contributions to $\kappa_{\mathrm{L}}$ as important as those from diagonal terms in the heat-flux operator. Finally, we discuss the limitations of our method and possible future directions.

\section{METHODS}

\section{A. Lattice thermal transport from the Peierls-Boltzmann approach and beyond}

The hierarchy of our theoretical model is built upon the Peierls-Boltzmann approach, wherein the contribution of phonons towards heat transfer is modeled [21]. The resulting $\kappa_{\mathrm{L}}$ has a straightforward expression under the single-mode relaxation time approximation (SMRTA):

$$
\kappa_{\mathrm{L}}=\frac{\hbar^{2}}{k_{B} T^{2} \Xi N} \sum_{\lambda} n_{\lambda}\left(n_{\lambda}+1\right) \omega_{\lambda}^{2} \mathbf{v}_{\lambda} \otimes \mathbf{v}_{\lambda} \tau_{\lambda},
$$

where $\hbar, k_{B}, T, \Xi$, and $N$ are, respectively, the reduced Planck constant, Boltzmann's constant, absolute temperature, volume of the primitive cell, and the number of sampled wave vectors. The phonon mode-resolved 
properties, namely, $n_{\lambda}, \omega_{\lambda}, \mathbf{v}_{\lambda}$, and $\tau_{\lambda}$, are the population, frequency, group velocity, and lifetime of mode $\lambda$ (composite index for the wave vector $\mathbf{q}$ and branch $s$ ), respectively. Normally, all phonon properties except $\tau_{\lambda}$ can be obtained from the harmonic approximation (HA) by the diagonalizing dynamical matrix constructed from harmonic interatomic force constants (IFCs). The calculation of $\tau_{\lambda}$ requires information beyond the HA and must account for at least 3ph scattering processes, which is usually performed by treating cubic anharmonicity as a perturbation to the HA $[10,11,85]$.

These approximations provide the basis for computing $\kappa_{\mathrm{L}}$ in the framework of ALD and PBTE, representing the lowest or first level of theory, hereinafter abbreviated as $\mathrm{HA}+3 \mathrm{ph}$ and $\kappa_{3 \mathrm{ph}}^{\mathrm{HA}}$ for the resultant $\kappa_{\mathrm{L}}$. The inclusion of quartic anharmonicity will affect both $\omega_{\lambda}$ and $\tau_{\lambda}$. The $\mathrm{HA}+3$ ph model can be improved by taking into account the phonon frequency shifts at finite temperature owing to APRN [13]. Among various existing formalisms for APRN [51,86-89], we choose the self-consistent phonon (SCPH) approximation, which comprises the first-order correction from quartic anharmonicity [90-93]. The resultant phonon frequencies, in principle, better represent the energies of phonon quasiparticles, particularly in the presence of severe anharmonicity and lattice instability [40,51,94]. We label this improved model the second level of theory, abbreviated as $\mathrm{SCPH}+3 \mathrm{ph}$ and $\kappa_{3 \mathrm{ph}}^{\mathrm{SCPH}}$. Finally, additional phonon scattering rates from $4 \mathrm{ph}$ scattering processes can be added on top of the 3 ph scattering rates [52], constituting the highest or third level of theory used in this study, namely, $\mathrm{SCPH}+3,4 \mathrm{ph}$ and $\kappa_{3,4 \mathrm{ph}}^{\mathrm{SCPH}}$. A schematic showing the ladder of approximations is depicted in Fig. 2(a), wherein the additional phonon scattering due to naturally occurring isotopes [95-97] is implicitly included in every ladder of approximations. We note that while it is not immediately obvious where phonon frequency shifts and scattering due to quartic anharmonicity should lie on our ladder of approximations, the order adopted here is based on the fact that scattering rates depend on frequencies, so better estimations of phonon frequencies should lead to more accurate scattering rates. It is worth noting that although the $\mathrm{HA}+3 \mathrm{ph}$ scheme is now commonplace, a partial consideration of quartic anharmonicity for either frequency shifts or scattering rates is limited to a few studies [40,44,51-53,57,94,98,99], and full consideration of quartic anharmonicity is rarely reported [45,46,54,56,100-104].

However, the above thermal transport models only capture the diagonal terms of the heat-flux operator [64], consistent with PGM. In fact, even in the harmonic part of the heat flux, there are off-diagonal terms that contribute additional heat transport, although their magnitude compared to the diagonal part ( $\left.\kappa_{\mathrm{L}}^{\text {diagonal }}\right)$ is usually deemed negligible in simple crystalline compounds $[64,105]$. The theoretical formalism for estimating the off-diagonal contributions $\left(\kappa_{\mathrm{L}}^{\text {off-diagonal }}\right)$ has been derived [106-108] and is often used to explain the glasslike $\kappa_{\mathrm{L}}$ in amorphous compounds [109]. Recently, a unified thermal transport model incorporating both diagonal and off-diagonal contributions considering anharmonic phonon-phonon interactions has been developed [65,110]. In this work, we adopt the formalism derived by Simoncelli, Marzari, and Mauri [65] to estimate $\kappa_{\mathrm{L}}^{\text {off-diagonal }}$ on top of the $\mathrm{SCPH}+3,4 \mathrm{ph}$ theory:

$$
\begin{aligned}
\kappa_{\mathrm{L}}^{\text {off-diagonal }}= & \frac{\hbar^{2}}{k_{B} T^{2} \Xi N} \sum_{\mathbf{q}} \sum_{s \neq s^{\prime}} \frac{\omega_{\mathbf{q}}^{s}+\omega_{\mathbf{q}}^{s^{\prime}}}{2} \mathbf{v}_{\mathbf{q}}^{s, s^{\prime}} \otimes \mathbf{v}_{\mathbf{q}}^{s^{\prime}, s} \\
& \times \frac{\omega_{\mathbf{q}}^{s} n_{\mathbf{q}}^{s}\left(n_{\mathbf{q}}^{s}+1\right)+\omega_{\mathbf{q}}^{s^{\prime}} n_{\mathbf{q}}^{s^{\prime}}\left(n_{\mathbf{q}}^{s^{\prime}}+1\right)}{4\left(\omega_{\mathbf{q}}^{s^{\prime}}-\omega_{\mathbf{q}}^{s}\right)^{2}+\left(\Gamma_{\mathbf{q}}^{s}+\Gamma_{\mathbf{q}}^{s^{\prime}}\right)^{2}}\left(\Gamma_{\mathbf{q}}^{s}+\Gamma_{\mathbf{q}}^{s^{\prime}}\right) .
\end{aligned}
$$

Note that the phonon lifetime $\tau_{\lambda}$ is replaced with the scattering rate $\Gamma_{\mathbf{q}}^{s}=1 / \tau_{\lambda}$. In addition, the group velocity is generalized to the off-diagonal form $\mathbf{v}_{\mathbf{q}}^{s, s^{\prime}}$ [see Eq. (30) in Ref. [65] and Eq. (4) in Ref. [111]].

We have adopted a full solution of PBTE using an iterative scheme $[112,113]$ beyond the SMRTA to treat the nonresistive normal scattering processes when only $3 \mathrm{ph}$ scattering is considered, taking advantage of the existing implementations within the ShengBTE package [114]. The evaluation of the SCPH equation, $4 \mathrm{ph}$ scattering rates, and the off-diagonal part of $\kappa_{\mathrm{L}}$ were performed using an inhouse implementation of these schemes. As a compromise between computational cost and accuracy, we calculated 4 ph scattering rates under SMRTA and added them to the iteratively solved $3 \mathrm{ph}$ scattering rates following Matthiessen's rule, as adopted in an earlier study [45]. This treatment certainly has limitations and tends to potentially underestimate $\kappa_{\mathrm{L}}$, which will be discussed in Sec. III C. Phonon wave vectors were sampled with a uniform $16 \times 16 \times 16$ q-point mesh for both Eqs. (1) and (2), and with a sparser $8 \times 8 \times 8$ q-point mesh for $\mathrm{SCPH}$ calculations. These choices were made based on rigorous convergence tests, consistent with the parameters used in earlier studies $[44,45,54,57,115]$. We refer the readers to the appendices for more details on solving the $\mathrm{SCPH}$ equation (Appendix A), numerical evaluation of phonon scattering rates (Appendix $\mathrm{B}$ ), and construction of high-order IFCs using CSLD (Appendix C).

\section{B. Density functional theory calculations}

All DFT calculations were performed using the Vienna Ab initio/ Simulation Package (VASP) [116-119], which employs the projector-augmented wave (PAW) [120] method in conjunction with the Perdew-Burke-Ernzerhof (PBE) [121] version of the generalized gradient 
approximation (GGA) [122] for the exchange-correlation functional [14], unless otherwise noted. We used Gammacentered $\mathbf{k}$-point meshes with the smallest allowed spacing of $0.15 \AA^{-1}$ between $\mathbf{k}$ points, a plane-wave cutoff energy at least 30\% higher than the "ENMAX" values specified in the recommended PAW pseudopotentials by VASP. Atomic and cell degrees of freedom were taken into account in all structural relaxations, with the convergence thresholds of $10^{-3} \mathrm{eV} / \AA$ for force components and $10^{-8} \mathrm{eV}$ for total energy, respectively. Born effective charges and macroscopic static dielectric constants used for a nonanalytic correction of phonon dispersions [123] near the Gamma point were computed using the density function perturbation theory (DFPT) implementation in VASP [124,125]. All calculations, including structural relaxation and symmetrization of a primitive cell, construction of supercells, and self-consistent force calculations of the supercells with displaced atoms (for extracting IFCs), were performed using our recently implemented high-throughput phonons framework within the Open Quantum Materials Database (OQMD) [126,127]. A full account of this framework will be given in a separate publication [60].

\section{RESULTS AND DISCUSSIONS}

\section{A. Lattice thermal conductivity}

We start our discussion of the $\kappa_{\mathrm{L}}$ of the 37 binary compounds listed in Table I by analyzing $\kappa_{\mathrm{L}}$, which is obtained using the lowest level of theory, namely, $\mathrm{HA}+3 \mathrm{ph}$. Since the $\kappa_{\mathrm{L}}$ values span about 3 orders of magnitude, we show the ratio of $\kappa_{3 \mathrm{ph}}^{\mathrm{HA}}$ to $\kappa_{\mathrm{L}}^{\mathrm{exp}}$ as a function of $\kappa_{\mathrm{L}}^{\exp }$ (all at $300 \mathrm{~K}$ ) in Fig. 1 to better present a comparison with experiments. We observe several interesting features: (i) $\kappa_{3 \mathrm{ph}}^{\mathrm{HA}}$ of rocksalts generally underestimates $\kappa_{\mathrm{L}}$, while $\kappa_{3 \mathrm{ph}}^{\mathrm{HA}}$ of zinc blendes, on the contrary, tends to overestimate $\kappa_{\mathrm{L}}$, and (ii) several compounds, e.g., $\mathrm{HgSe} / \mathrm{Te}, \mathrm{BAs}, \mathrm{PbS}$, and $\mathrm{KI}$, in both rocksalt and zinc blende families deviate significantly from experiments. Our observation (i) is consistent with an earlier study by Seko et al. [128], albeit the focus therein was on predicting low- $\kappa_{\mathrm{L}}$ systems using Bayesian optimization and not on the analysis of the differences between theory and experiment. The discrepancies may be attributed to several sources such as uncertainties in experimental sample preparation and measurement, the fundamental limitations of DFT (e.g., approximations to the exchange correlation functional), the theoretical model adopted in computing $\kappa_{\mathrm{L}}$, and so on. However, the contrasting behaviors (systematic underestimation vs overestimation) in rocksalts and zinc blendes are unlikely to be attributable to either experimental uncertainties or the approximations used in DFT calculations. If the discrepancies were due to either of these, the uncertainties associated with both cases would likely be systematic across these compounds. For example, if the PBE functional adopted in this study tends to underestimate

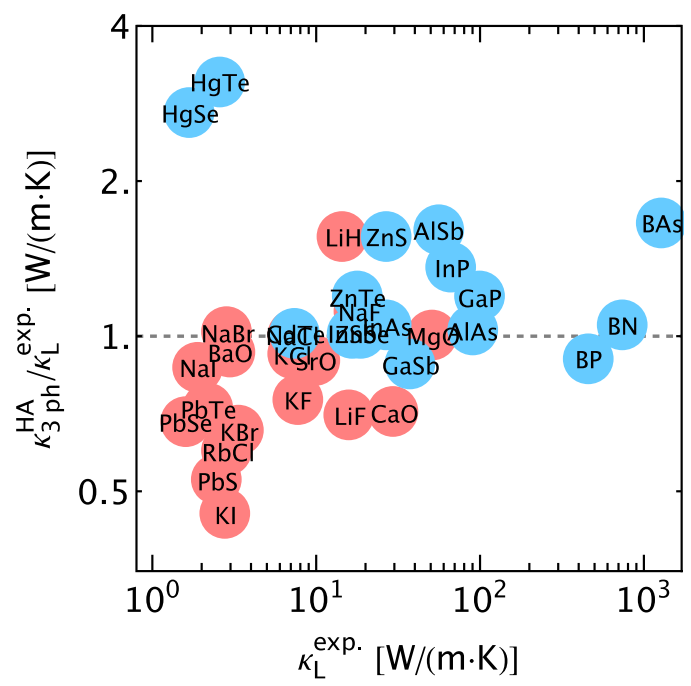

FIG. 1. Comparison of theoretically calculated $\left(\kappa_{3 \mathrm{ph}}^{\mathrm{HA}}\right)$ and experimentally measured $\left(\kappa_{\mathrm{L}}^{\mathrm{exp}}\right)$ lattice thermal conductivities in selected binary rocksalts (red disks) and zinc blendes (blue disks) at $300 \mathrm{~K}$. The theoretical values are calculated at the level of $\mathrm{HA}+3 \mathrm{ph}$, namely, phonon dispersion from the HA and phonon scattering rates from $3 \mathrm{ph}$ scattering processes. The ratio of $\kappa_{3 \mathrm{ph}}^{\mathrm{HA}} / \kappa_{\mathrm{L}}^{\mathrm{exp}}$ is calculated by dividing the theoretical value by the higher end of the experimental values, both listed in Table I.

binding energies and thereby bond strength, the calculated $\kappa_{\mathrm{L}}$ of rocksalts and zinc blendes, both extended solids, using the PBE functional should be underestimated. On the other hand, experimentally measured $\kappa_{\mathrm{L}}$ of polycrystalline compounds would exhibit consistently lower $\kappa_{\mathrm{L}}$ than those from single crystals. A critical question then arises: Can such discrepancies be systematically reduced by improving the theoretical model for computing $\kappa_{\mathrm{L}}$ ?

We proceed to answer this question by examining the role of quartic anharmonicity in determining $\kappa_{\mathrm{L}}$. We progressively climb our ladder of approximations, refining the HA +3 ph model by accounting for phonon frequency shifts and scattering rates from quartic anharmonicity into two improved models, namely, $\mathrm{SCPH}+3 \mathrm{ph}$ and $\mathrm{SCPH}+3,4 \mathrm{ph}$. We show the calculated $\kappa_{3 \mathrm{ph}}^{\mathrm{SCPH}}$ and $\kappa_{3,4 \mathrm{ph}}^{\mathrm{SCPH}}$, both divided by $\kappa_{3 \mathrm{ph}}^{\mathrm{HA}}$, to better illustrate the relative change in $\kappa_{\mathrm{L}}$ in Figs. 2(b) and 2(c) for rocksalts and zinc blendes, respectively. From the calculated ratio $\kappa_{3 \mathrm{ph}}^{\mathrm{SCPH}} / \kappa_{3 \mathrm{ph}}^{\mathrm{HA}}$, it is evident that the effects of APRN on $\kappa_{\mathrm{L}}$ are quite different for these two classes of materials: While APRN tends to increase $\kappa_{\mathrm{L}}$ in rocksalts, it has a much weaker impact (a very slight reduction) on $\kappa_{\mathrm{L}}$ in zinc blendes, and exceptions are only found for $\mathrm{HgS} / \mathrm{Se} / \mathrm{Te}$ whose $\kappa_{\mathrm{L}}$ are slightly enhanced. The increase in $\kappa_{\mathrm{L}}$ in some rocksalts is quite significant, e.g., in $\mathrm{BaO}, \mathrm{NaI}, \mathrm{KI}$, and $\mathrm{PbS} / \mathrm{Se} / \mathrm{Te}$, revealing the importance of APRN due to finite-temperature effects. With additional $4 \mathrm{ph}$ scattering, as indicated by the ratio $\kappa_{3,4 \mathrm{ph}}^{\mathrm{SCPH}} / \kappa_{3 \mathrm{ph}}^{\mathrm{HA}}$, the $\kappa_{\mathrm{L}}$ for all compounds is reduced, by various amounts, due to shortened phonon lifetimes. By 
(a)

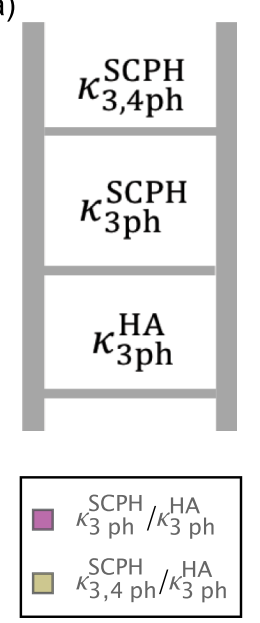

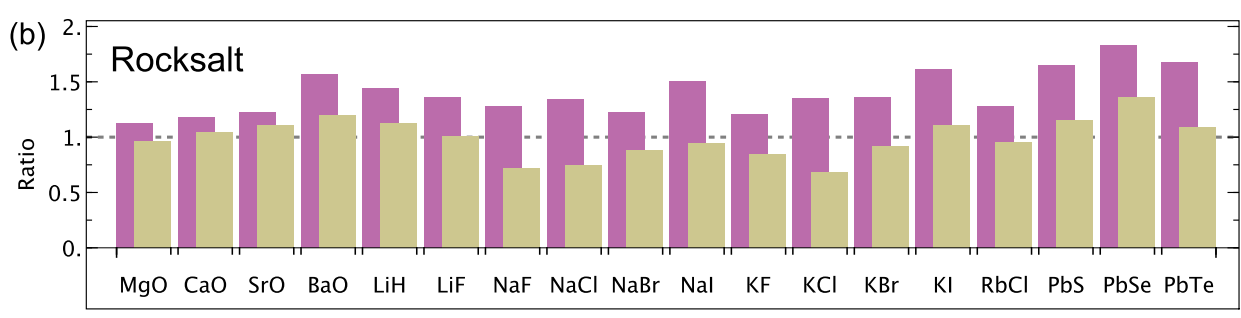

(c) $2 .-F$ Zincblende

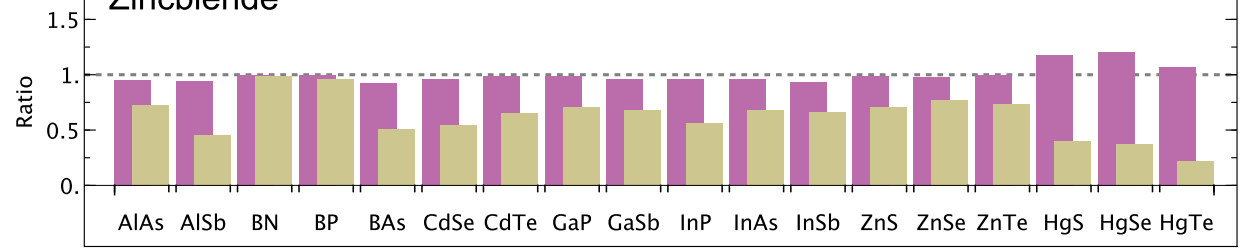

FIG. 2. Comparison of lattice thermal conductivity calculated using various levels of theories for selected binary rocksalts and zinc blendes at $300 \mathrm{~K}$. (a) Schematic showing the ladder of approximations employed in computing $\kappa_{\mathrm{L}}$ based on the phonon gas model, namely, $\mathrm{HA}+3 \mathrm{ph}, \mathrm{SCPH}+3 \mathrm{ph}$, and $\mathrm{SCPH}+3,4 \mathrm{ph}$, denoting the level of theory from low to high. HA and SCPH, respectively, denote the harmonic approximation (without anharmonic renormalization) and self-consistent phonon approximation (with anharmonic renormalization from quartic anharmonicity) used for computing phonon frequencies, and 3ph and 4ph indicate three-phonon and fourphonon scattering rates, respectively. The resulting lattice thermal conductivities are denoted as $\kappa_{3 \mathrm{ph}}^{\mathrm{HA}}, \kappa_{3 \mathrm{ph}}^{\mathrm{SCPH}}$, and $\kappa_{3,4 \mathrm{ph}}^{\mathrm{SCPH}}$, respectively. (b,c) Calculated ratio of $\kappa_{3 \mathrm{ph}}^{\mathrm{SCP}} / \kappa_{3 \mathrm{ph}}^{\mathrm{HA}}$ (purple bars) and $\kappa_{3,4 \mathrm{ph}}^{\mathrm{SCPH}} / \kappa_{3 \mathrm{ph}}^{\mathrm{HA}}$ (yellow bars), respectively. The absolute values of $\kappa_{3 \mathrm{ph}}^{\mathrm{HA}}, \kappa_{3 \mathrm{ph}}^{\mathrm{SCPH}}$, and $\kappa_{3,4 \mathrm{ph}}^{\mathrm{SCPH}}$ are listed in Table I.

comparing $\kappa_{3 \mathrm{ph}}^{\mathrm{HA}}$ and $\kappa_{3,4 \mathrm{ph}}^{\mathrm{SCPH}}$, we notice a significant reduction of $\kappa_{\mathrm{L}}$ in BAs and $\mathrm{AlSb}$, consistent with earlier findings $[44,57]$. In addition, we identify several previously unreported cases where the reduction due to 4 ph scattering is striking - CdSe, InP, and $\mathrm{HgS} / \mathrm{Se} / \mathrm{Te}$.

The net effect of quartic anharmonicity, including both APRN and 4ph scattering, is highly nontrivial: 4ph interactions always suppress $\kappa_{\mathrm{L}}$ in zinc blendes but can either increase or decrease $\kappa_{\mathrm{L}}$ in rocksalts depending on the system. In addition, some chemical trends emerge: In $\mathrm{Mg} / \mathrm{Ca} / \mathrm{Sr} / \mathrm{BaO}, \mathrm{NaF} / \mathrm{Cl} / \mathrm{Br} / \mathrm{I}$, and $\mathrm{KCl} / \mathrm{Br} / \mathrm{I}$, the ratio $\kappa_{3,4 \mathrm{ph}}^{\mathrm{SCPH}} / \kappa_{3 \mathrm{ph}}^{\mathrm{HA}}$ tends to increase with the size of the cation/anion. In the BN/P series, both APRN and 4ph scattering have little effect, in striking contrast to BAs. We note that although using a different exchange-correlation functional is expected to affect the absolute values of $\kappa_{3 \mathrm{ph}}^{\mathrm{HA}}, \kappa_{3 \mathrm{ph}}^{\mathrm{SCPH}}$, and $\kappa_{3,4 \mathrm{ph}}^{\mathrm{SCPH}}$, the presented ratios $\kappa_{3}^{\mathrm{SCPH}} / \kappa_{3 \mathrm{ph}}^{\mathrm{HA}}$ and $\kappa_{3,4 \mathrm{ph}}^{\mathrm{SCPH}} / \kappa_{3 \mathrm{ph}}^{\mathrm{HA}}$ are expected to be qualitatively robust due to cancellation of errors.

\section{B. Phonon frequency shifts}

Following the contrasting impact of APRN on $\kappa_{\mathrm{L}}$ in rocksalts and zinc blendes, we investigate the shifts in phonon frequencies and their effect on the calculated $\kappa_{\mathrm{L}}$. Figure 3(a) shows the comparison of the Frobenius norm of the shifts in phonon frequencies $\|\Delta \omega\|_{F}$ in rocksalts and zinc blendes as a function of the Frobenius norm of the phonon frequencies $\|\omega\|_{F}$. Both $\|\Delta \omega\|_{F}$ and $\|\omega\|_{F}$ are calculated using the phonon modes associated with an $8 \times 8 \times 8 \mathbf{q}$-mesh and normalized by the total number of phonon modes. We notice that (i) the relative frequency shift $\left(\|\Delta \omega\|_{F} /\|\omega\|_{F}\right)$ decreases with increasing $\|\omega\|_{F}$, and (ii) the relative frequency shifts in rocksalts are almost 1 order of magnitude larger than those in zinc blendes. Observation (i) is consistent with the fact that compounds with higher harmonic phonon frequencies generally have weaker anharmonicity and thus smaller frequency shifts from APRN. Observation (ii) helps explain the distinct behaviors of rocksalts and zinc blendes in terms of the difference between $\kappa_{3 \mathrm{ph}}^{\mathrm{SCPH}}$ and $\kappa_{3 \mathrm{ph}}^{\mathrm{HA}}$, observed in Sec. III A and seen in Fig. 2. In other words, relatively small frequency shifts only change $\kappa_{\mathrm{L}}$ slightly in zinc blendes (corresponding to $\kappa_{3 \mathrm{ph}}^{\mathrm{SCPH}} / \kappa_{3 \mathrm{ph}}^{\mathrm{HA}} \approx 1$ ), while the much larger frequency shifts in rocksalts (an order of magnitude higher than in zinc blendes) lead to a significant change in $\kappa_{\mathrm{L}}$. An outlier among the zinc blendes is the $\mathrm{HgS} / \mathrm{Se} / \mathrm{Te}$ family, which has the largest frequency shifts and correspondingly large enhancement in $\kappa_{\mathrm{L}}$ upon including APRN. The increasing trend in $\kappa_{3 \mathrm{ph}}^{\mathrm{SCPH}} / \kappa_{3 \mathrm{ph}}^{\mathrm{HA}}$ among $\mathrm{Mg} / \mathrm{Ca} / \mathrm{Sr} / \mathrm{BaO}$ can also be understood to result almost entirely from the relative shifts in phonon frequencies. In addition, we find that the frequency shifts in rocksalts are mostly positive, while they are slightly negative in zinc blendes, except $\mathrm{HgS} / \mathrm{Se} / \mathrm{Te}$ [Fig. 3(a) only shows magnitudes due to the usage of Frobenius norm], consistent with the slightly decreased $\kappa_{\mathrm{L}}$ of the latter.

To better illustrate the different behaviors exhibited in rocksalts and zinc blendes, we present a detailed study 

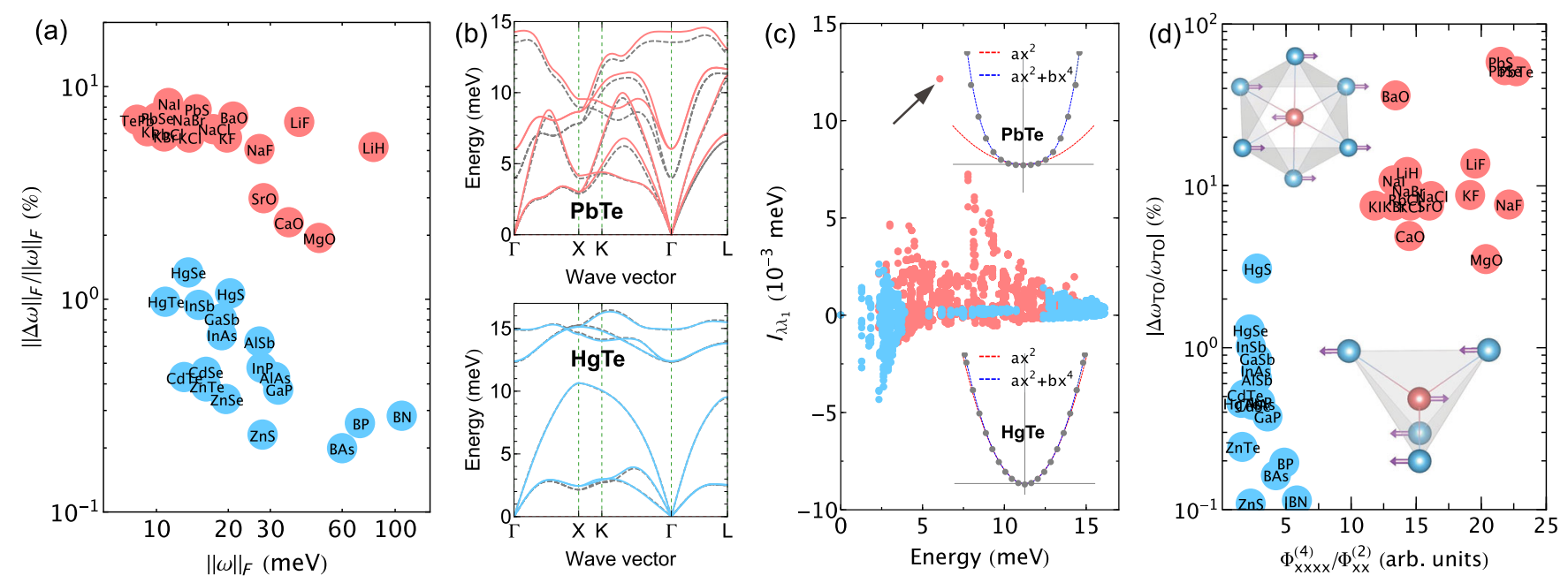

FIG. 3. Phonon frequency shifts when including quartic anharmonicity in selected binary rocksalts (red disks) and zinc blendes (blue disks). (a) Ratio of the Frobenius norm of the shifts in phonon frequencies $\|\Delta \omega\|_{F}$ to the Frobenius norm of the phonon frequencies $\|\omega\|_{F}$ as a function of $\|\omega\|_{F}$. The self-consistent phonon calculations including quartic anharmonicity are performed at $300 \mathrm{~K}$. (b) Phonon dispersions of rocksalt PbTe (upper panel) and zinc blende HgTe (lower panel). The dashed gray lines denote the phonon dispersion from the harmonic approximation, and the colored solid lines denote the phonon dispersion including APRN at $300 \mathrm{~K}$. (c) Strength of the four-phonon interaction matrix elements $\left(I_{\lambda \lambda_{1}}\right)$ associated with the zone-center transverse optical (TO) phonon mode in the self-consistent phonon formalism (definition in Appendix A) as a function of phonon frequency for PbTe (red dots) and HgTe (blue dots). The black arrow points to the largest value of $I_{\lambda \lambda_{1}}$ in PbTe, with both $\lambda$ and $\lambda_{1}$ denoting the TO phonon mode. The insets show the PES of the zone-center TO mode for PbTe (upper) and HgTe (lower), respectively. The DFT computed energies are denoted by disks, while the fitted PES using polynomials up to second and fourth order are denoted by red and blue dashed lines, respectively. (d) Ratio of the shift in the frequency of the zone-center TO phonon mode $\left(\Delta \omega_{\mathrm{TO}}\right)$ to the harmonic value $\left(\omega_{\mathrm{TO}}\right)$ as a function of the ratio of quartic on-site interatomic force constants (IFCs) to harmonic IFCs along the $x$ axis. The insets show the atomic displacement vectors associated with the zone-center TO mode for rocksalts (upper) and zinc blendes (lower), respectively.

of rocksalt $\mathrm{PbTe}$ and zinc blende $\mathrm{HgTe}$ as representative cases - they have similar atomic masses and charge states, and are both promising for thermoelectric applications $[129,130]$. The calculated phonon dispersions with and without APRN for the two compounds are shown in Fig. 3(b). We find that the phonon dispersion of $\mathrm{HgTe}$ changes little after including APRN, but there is a substantial change in that of $\mathrm{PbTe}$, particularly for the $\mathrm{TO}$ phonon modes. We note that the significant hardening of the zone-center TO mode from about $4 \mathrm{meV}$ to $6 \mathrm{meV}$ at $300 \mathrm{~K}$ in $\mathrm{PbTe}$ has been reported in several earlier studies that take into account finite-temperature effects $[36,45,131,132]$, consistent with our SCPH calculations in this work. It should be emphasized that a direct comparison of the calculated dispersion with experimental measurement should be done with caution, as phonon frequencies are also affected by other phonon softening mechanisms such as thermal expansion. We did not include thermal expansion universally in this work because we find that, for example, in the cases of PbTe of $\mathrm{HgTe}$, the GGA-PBE functional already predicts underestimated phonon frequencies of optical modes [133,134], and including additional thermal expansion further softens phonons, which is expected to result in a large deviation from experimental measurements. Moreover, second-order effects from 3ph interactions, which are not considered in this study and probably can be neglected in highsymmetry cubic phases [87], will lead to additional frequency shifts that are fully frequency dependent $[12,13]$. The large phonon frequency shifts due to anharmonic phonon-phonon interactions (as in the case of binary rocksalts) imply a qualitative breakdown of the quasiharmonic approximation (QHA) because it only accounts for volume dependence of the phonon frequencies.

To reveal the microscopic origin of the strikingly different shifts in phonon frequencies in $\mathrm{PbTe}$ and $\mathrm{HgTe}$, we show in Fig. 3(c) the 4ph interaction matrix element $\left(I_{\lambda \lambda_{1}}\right.$; see Appendix A) associated with the zone-center TO mode in the SCPH formalism. Physically, the net shift in frequency of the zone-center TO mode $(\lambda)$ is a cumulative effect of its interactions with all the other phonon modes $\left(\lambda_{1}\right)$, which could either soften or harden the TO mode depending on the coupling coefficients, as indicated by the widely distributed dots with both positive and negative values in Fig. 3(c). What makes PbTe different from $\mathrm{HgTe}$ is that the distribution of $I_{\lambda \lambda_{1}}$ in $\mathrm{PbTe}$ is quite asymmetric around zero values and exhibits mostly positive values, thus leading to an appreciable net-positive shift frequency. On the contrary, the distribution of $I_{\lambda \lambda_{1}}$ in $\mathrm{HgTe}$ is quite symmetric, which results in nearly a vanishing net shift in 
frequency. It is noteworthy that the largest $I_{\lambda \lambda_{1}}$ in $\mathrm{PbTe}$ among all pairwise interactions of phonon modes $\lambda$ and $\lambda_{1}$, as pointed out by the arrow in Fig. 3(c), is the one in which the zone-center TO mode interacts with itself; the corresponding interaction in $\mathrm{HgTe}$ is vanishingly small. Physically, when $\lambda$ equals $\lambda_{1}, I_{\lambda \lambda_{1}}$ can be interpreted in the frozen phonon picture of the zone-center TO mode. Therefore, large $I_{\lambda \lambda}$ indicates a strong quartic contribution to the PES when atoms move in accordance with the displacement pattern of the zone-center TO mode.

This interpretation in terms of the zone-center TO mode is confirmed by the PES of the mode in PbTe and $\mathrm{HgTe}$ [the insets of Fig. 3(c)] - there is a strong effect of the quartic terms in $\mathrm{PbTe}$, while the quadratic term dominates in $\mathrm{HgTe}$. An intuitive interpretation of phonon hardening is that atoms at higher temperatures experience large displacements and explore the higher-order PES, which repels them and thus enhances the effective phonon frequency. Note that we have transformed the understanding of the frequency shift in reciprocal space to the shape of PES in real space. This approach encourages us to examine the correlation between the frequency shift of the zone-center TO mode and the strength of quartic anharmonicity relative to the harmonic IFCs [i.e., $\Phi^{(4)} / \Phi^{(2)}$; shown in Fig. 3(d)]. It is clear that the ratio $\Phi^{(4)} / \Phi^{(2)}$ in zinc blendes is, overall, much smaller than that in rocksalts, well correlated with the relative frequency shift of the zone-center TO mode. The much larger $\Phi^{(4)} / \Phi^{(2)}$ in rocksalts when compared to zinc blendes may be directly attributed to the different coordination environments (octahedral vs tetrahedral, respectively) in their crystal structures. We propose that the octahedral coordination, in general, allows for larger atomic displacements than a tetrahedral coordination environment. Thus, (i) for small displacements, the atoms feel relatively weak repelling forces from the harmonic PES, but (ii) for vibrations further away from the equilibrium position, the atoms feel strong repelling forces from the quartic PES, somewhat resembling the behavior of rattling atoms in a cagelike structure. To partially confirm this conjecture, we calculate the PES of the zone-center TO mode of PbTe in a hypothetical zinc blende structure and find the PES resembles that of $\mathrm{HgTe}$ and shows a significantly reduced quartic contribution. This conjecture may be further tested in other systems containing these two distinctive coordination environments.

\section{Phonon scattering phase space}

To illustrate the correlation between the phonon frequency shifts and the resulting changes in $\kappa_{\mathrm{L}}$, we show the $\kappa_{3 \mathrm{ph}}^{\mathrm{SCPH}} / \kappa_{3 \mathrm{ph}}^{\mathrm{HA}}$ as a function of $\|\Delta \omega\|_{F} /\|\omega\|_{F}$ in Fig. 4(a). It can be seen that there is a strong positive correlation between $\kappa_{3 \mathrm{ph}}^{\mathrm{SCH}} / \kappa_{3 \mathrm{ph}}^{\mathrm{HA}}$ and $\|\Delta \omega\|_{F} /\|\omega\|_{F}$. The zinc blendes are well separated from the rocksalts because of small values of $\|\Delta \omega\|_{F} /\|\omega\|_{F}$ and $\kappa_{3 \mathrm{ph}}^{\mathrm{SCPH}} / \kappa_{3 \mathrm{ph}}^{\mathrm{HA}}$ approaching unity, except $\mathrm{HgS} / \mathrm{Se} / \mathrm{Te}$. For the rocksalts, aside from $\mathrm{Mg} / \mathrm{Ca} /$ $\mathrm{SrO}$, all the others have values of $\|\Delta \omega\|_{F} /\|\omega\|_{F}$ within a range of $5 \%$ to $9 \%$, giving rise to a roughly $40 \%$ increase in $\kappa_{3 \mathrm{ph}}^{\mathrm{SCPH}}$ compared to $\kappa_{3 \mathrm{ph}}^{\mathrm{HA}}$. The large spread of $\kappa_{3 \mathrm{ph}}^{\mathrm{SCPH}} / \kappa_{3 \mathrm{ph}}^{\mathrm{HA}}$ for a given value of $\|\Delta \omega\|_{F} /\|\omega\|_{F}$ may be traced back to the subtle impact of a mode-wise frequency shift on $\kappa_{\mathrm{L}}$ among different compounds, which are smeared out by using the Frobenius norms of $\Delta \omega$ and $\omega$. Improvements may be achieved by further accounting for the weights from modewise group velocity and heat capacity. Nevertheless, such a strong correlation unambiguously reveals the importance of APRN and the rather different behaviors in rocksalts and zinc blendes. Additionally, by separately examining the impacts of group velocity and heat capacity on $\kappa_{\mathrm{L}}$, we find the increment in $\kappa_{\mathrm{L}}$ is mostly attributable to the reduction in phonon scattering rates.

Here, we evaluate $3 \mathrm{ph}$ and $4 \mathrm{ph}$ scattering rates in a bruteforce manner for all studied compounds, but for much more complex systems or large-scale calculations [135-138], the computational cost would be formidable even for $3 \mathrm{ph}$ scattering, not to mention the $4 \mathrm{ph}$ scattering, of which the computational cost is extremely large. Then, a key question arises as to whether we can achieve a qualitative estimate via much cheaper calculations within the harmonic approximation. Earlier studies of the phonon scattering phase space $(\Theta$; see Appendix B)—one of the key quantities that determine phonon scattering rates-showed promise as an effective indicator for the strength of phonon scattering [139]. We are thus motivated to investigate the correlation between $\kappa_{\mathrm{L}}$ and $\Theta$; the latter accounts for all the other factors, except $3 \mathrm{ph} / 4 \mathrm{ph}$ interaction matrix elements in evaluating scattering rates, and it is readily accessible from the harmonic phonon dispersion. Figure 4(b) plots $\kappa_{3 \mathrm{ph}}^{\mathrm{SCPH}} / \kappa_{3 \mathrm{ph}}^{\mathrm{HA}}$ as a function of $\Theta_{3 \mathrm{ph}}^{\mathrm{SCPH}} / \Theta_{3 \mathrm{ph}}^{\mathrm{HA}}$, which displays clear negative correlation. The sizable spread of points around the approximated linear relation might be attributable to (i) the anisotropy of frequency shifts in reciprocal space and (ii) the intricate role of $3 \mathrm{ph} / 4 \mathrm{ph}$ matrix elements, both of which may adjust the weight of a mode-wise contribution to $\kappa_{\mathrm{L}}$. Meanwhile, a remarkable assessment of the strength of $4 \mathrm{ph}$ scattering is revealed by the relation between $\kappa_{3,4 \mathrm{ph}}^{\mathrm{SCPH}} / \kappa_{3 \mathrm{ph}}^{\mathrm{SCPH}}$ and $\Theta_{4 \mathrm{ph}}^{\mathrm{SCPH}} / \Theta_{3 \mathrm{ph}}^{\mathrm{SCPH}}$, as shown in Fig. 4(c). The strong negative correlation between $\kappa_{3,4 \mathrm{ph}}^{\mathrm{SCPH}} / \kappa_{3 \mathrm{ph}}^{\mathrm{SCPH}}$ and $\Theta_{4 \mathrm{ph}}^{\mathrm{SCPH}} / \Theta_{3 \mathrm{ph}}^{\mathrm{SCPH}}$ can explain (i) the vastly different impacts of $4 \mathrm{ph}$ scattering on $\kappa_{\mathrm{L}}$ among BN/P/As and (ii) the giant $4 \mathrm{ph}$ scattering in $\mathrm{HgS} / \mathrm{Se} / \mathrm{Te}$. This observation is encouraging because explicit evaluation of $4 \mathrm{ph}$ scattering rates is much more expensive than $3 \mathrm{ph}$ scattering (about $10^{3}$ to $10^{4}$ more CPU hours). With this established negative correlation, we may be able to better estimate $\kappa_{\mathrm{L}}$ by calculating only $3 \mathrm{ph}$ scattering rates and $4 \mathrm{ph}$ scattering phase space, without resorting to the cumbersome fourth-order IFCs at all. Moreover, the qualitative impact of 4 ph scattering relative to 3 ph scattering might be 

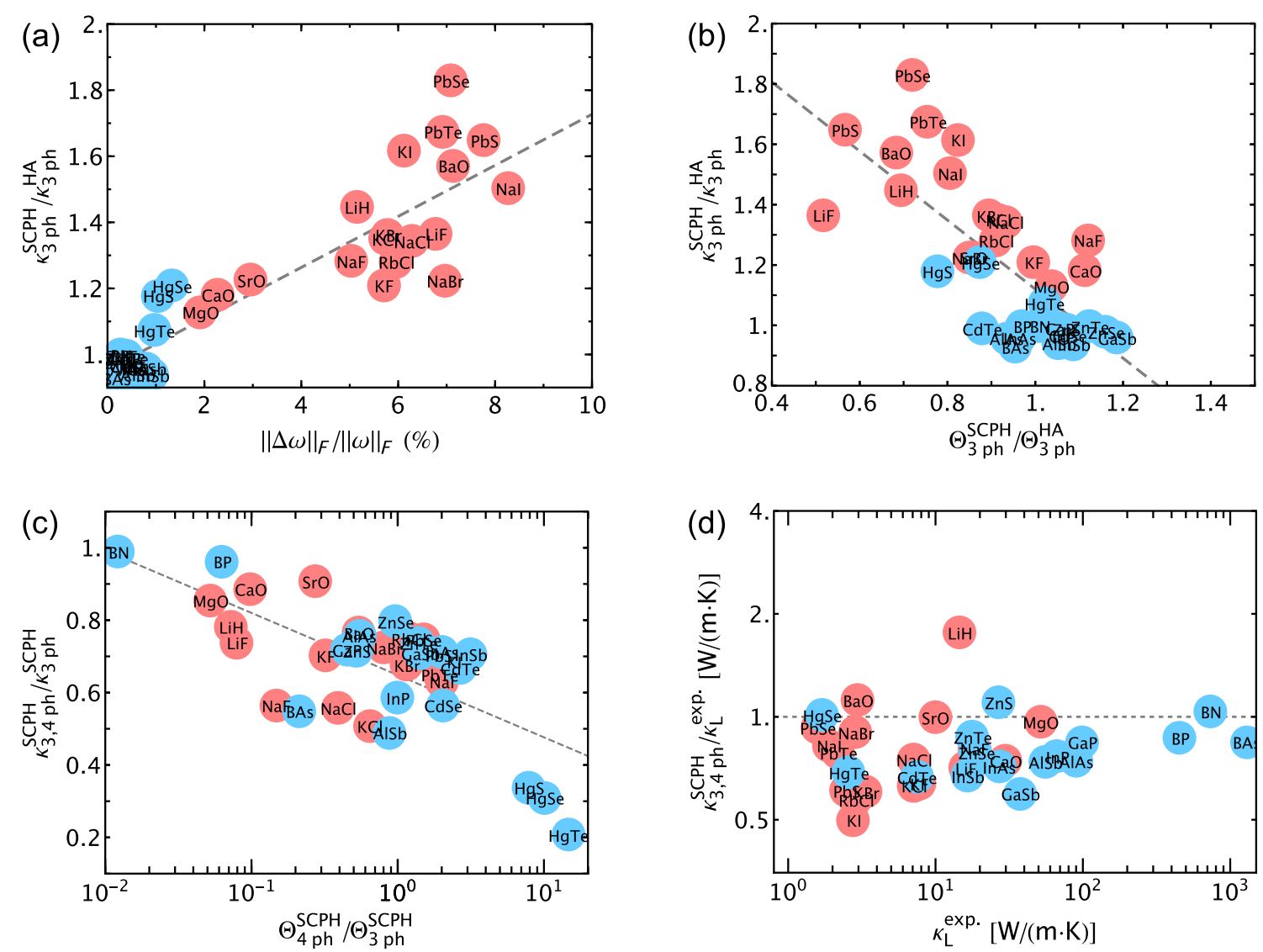

FIG. 4. Correlation between the lattice thermal conductivity and the phonon scattering phase space for the selected binary rocksalts (red disks) and zinc blendes (blue disks). (a) Ratio of $\kappa_{3 \mathrm{ph}}^{\mathrm{SCPH}}$ to $\kappa_{3 \mathrm{ph}}^{\mathrm{HA}}$ plotted as a function of the ratio $\|\Delta \omega\|_{F} /\|\omega\|_{F}$. (b) Ratio of $\kappa_{3 \mathrm{ph}}^{\mathrm{SCPH}}$ to $\kappa_{3 \mathrm{ph}}^{\mathrm{HA}}$ plotted as a function of the ratio of $\Theta_{3 \mathrm{ph}}^{\mathrm{SCPH}}$ to $\Theta_{3 \mathrm{ph}}^{\mathrm{HA}}$, wherein $\Theta$ denotes phonon scattering space (see Appendix B for the definition). (c) Ratio of $\kappa_{3,4 \mathrm{ph}}^{\mathrm{SCPH}}$ to $\kappa_{3 \mathrm{ph}}^{\mathrm{SCPH}}$ plotted as a function of the ratio of $\Theta_{4 \mathrm{ph}}^{\mathrm{SCPH}}$ to $\Theta_{3 \mathrm{ph}}^{\mathrm{SCPH}}$. (d) Ratio of $\kappa_{3,4 \mathrm{ph}}^{\mathrm{SCPH}}$ to $\kappa_{\mathrm{L}}^{\mathrm{exp}}$ plotted as a function of experimental lattice thermal conductivity $\kappa_{\mathrm{L}}^{\mathrm{exp}}$. The ratio $\kappa_{3,4 \mathrm{ph}}^{\mathrm{SCPH}} / \kappa_{\mathrm{L}}^{\mathrm{exp}}$ is obtained by dividing the theoretical value by the highest experimental value, both listed in Table I. The gray dashed lines in panels (a)-(c) represent the linear fit of the data, in line with the coordinate system, while in panel (d), the line denotes equal values between theory and experiment. All calculations are performed at $300 \mathrm{~K}$.

estimated by calculating only the scattering phase space using the harmonic phonon dispersion. Training a multifidelity machine-learning model to go from the harmonic 3 ph approximation to the anharmonic 3,4ph approximation accuracy for many compounds might also be feasible by taking advantage of the existing phonon databases $[135,140]$. We note that, as adopted throughout this study, correlating the relative change in both $\kappa_{\mathrm{L}}$ and $\Theta$ instead of their absolute values has the advantage of eliminating the system-dependent effects, such as the absolute magnitudes of phonon frequencies and $\kappa_{\mathrm{L}}$.

We compare the calculated $\kappa_{\mathrm{L}}$ with a full treatment of quartic anharmonicity to the experimental $\kappa_{\mathrm{L}}$ in Fig. 4(d). In contrast to the results presented in Fig. 1, we find that the discrepancy between the theoretical $\kappa_{\mathrm{L}}$ and the experimental $\kappa_{\mathrm{L}}$ is significantly reduced, with the root-mean-square error of their ratios $\left(\kappa_{3 \mathrm{ph}}^{\mathrm{HA}} / \kappa_{\mathrm{L}}^{\mathrm{exp}}\right.$ vs $\left.\kappa_{3,4 \mathrm{ph}}^{\mathrm{SCPH}} / \kappa_{\mathrm{L}}^{\exp }\right)$ relative to 1 decreasing from 0.55 to 0.29 . Moreover, we see that $\kappa_{3,4 \mathrm{ph}}^{\mathrm{SCPH}} / \kappa_{\mathrm{L}}^{\exp }$ of most compounds is now near or below 1 and has a mean value of 0.81 . Therefore, it is convincing that employing high-level theory can improve the predicted $\kappa_{\mathrm{L}}$ by reducing both deviation and variation, revealing the essential role of quartic anharmonicity. For the peculiar case of LiH whose $\kappa_{3,4 \mathrm{ph}}^{\mathrm{SCPH}} / \kappa_{\mathrm{L}}^{\text {exp }}$ stays way above 1 , we find that including thermal expansion reduces $\kappa_{3,4 \mathrm{ph}}^{\mathrm{SCPH}} / \kappa_{\mathrm{L}}^{\mathrm{exp}}$, making it closer to 1 (see Table I), which is consistent with the report in the literature that thermal expansion is rather important in $\mathrm{LiH}$ [141]. The relatively small overestimation of our theoretical value might be attributed to the absence of extrinsic phonon scatterings arising from defects and grain boundaries in our theoretical model. The effect of thermal expansion, which usually softens phonons, but is only considered here for several representative cases including $\mathrm{LiH}, \mathrm{PbTe}$, and $\mathrm{AgCl}$, may lead to an additional decrease in calculated $\kappa_{\mathrm{L}}$, albeit to what extent varies 
among compounds. Concerning the systematic underestimation of $\kappa_{\mathrm{L}}$, there are several possible factors. First, the exchange correlation functional used in this study is approximated at the level of GGA-PBE, which is shown to typically underestimate bulk moduli and phonon frequencies [134], consistent with the systematically underestimated $\kappa_{\mathrm{L}}$. To partially confirm this suspicion, we perform additional calculations for $\mathrm{PbTe}$ and $\mathrm{HgTe}$ using the PBEsol functional [142], which is specially designed for an improved description of solids [142]. As shown in Table I, the resulting $\kappa_{3,4 \mathrm{ph}}^{\mathrm{SCPH}}$ of $\mathrm{PbTe}$ and $\mathrm{HgTe}$ are significantly increased and agree much better with experiments than those obtained with the PBE functional. A full account of the impact of an exchange correlation functional on $\kappa_{\mathrm{L}}$ should be explored in a future study. Second, we have solved the PBTE under SMRTA for the 4ph scattering. Such an approximation results in a potential underestimation of $\kappa_{\mathrm{L}}$, particularly when Umklapp scattering is not dominant over Normal scattering. This result is reflected in the case of BAs, which is known to attain a large portion of Normal scattering, and thus explains our calculated value of $\kappa_{\mathrm{L}}$, which is smaller than earlier studies that employed either an approximated or a rigorous iterative solution of the PBTE, including both $3 \mathrm{ph}$ and $4 \mathrm{ph}$ scattering [8,44]. When Umklapp processes dominate the $4 \mathrm{ph}$ scattering [57], our approximation seems to be reasonable. Lastly, although the phonon frequencies (or second-order IFCs) are anharmonically renormalized at finite temperatures, the third- and fourth-order IFCs used in the evaluation of 3ph/ $4 \mathrm{ph}$ scattering rates are those obtained at $0 \mathrm{~K}$ without renormalization. An improvement might be achieved by performing an ensemble average of these IFCs at finite temperature $[143,144]$, which may be realized by means of the stochastic self-consistent harmonic approximation [145] or the temperature-dependent effective potential method [146].

\section{Dominant four-phonon scattering in HgTe}

Contrary to the common belief that high-order phononphonon scattering is only relevant at high temperatures, our results reveal that $4 \mathrm{ph}$ scattering is crucial even at room temperature in most of the studied compounds, whose $\kappa_{\mathrm{L}}$ could range from ultrahigh to very low. Furthermore, the calculated $\kappa_{\mathrm{L}}$ of rocksalts using levels of theory from low $(\mathrm{HA}+3 \mathrm{ph})$ to high $(\mathrm{SCPH}+3,4 \mathrm{ph})$ implies a general existence of cancellation of errors if neither phonon frequency shifts nor scattering rates due to quartic anharmonicity are considered. Despite such cancellation of errors, which might result in accidentally good agreement in $\kappa_{\mathrm{L}}$ between theory and experiment, the correct physics is not recovered in the lower level theory, potentially leading to an erroneous assessment of the mode-wise contribution to $\kappa_{\mathrm{L}}$. Compared to rocksalts, the effect of quartic anharmonicity on $\kappa_{\mathrm{L}}$ of zinc blendes is largely attributable to the $4 \mathrm{ph}$ scattering, due to the rather weak APRN. Among the studied zinc blendes, the $\mathrm{HgS} / \mathrm{Se} / \mathrm{Te}$ family is of particular interest because they exhibit the strongest reduction of $\kappa_{\mathrm{L}}$ due to 4 ph scattering among all the currently known or calculated compounds at $300 \mathrm{~K}$.

To gain a better understanding of their lattice dynamics and thermal transport properties, we proceed to discuss in detail the representative case $\mathrm{HgTe}$. We use both the PBE and the PBEsol functionals to compute phonon dispersions and compare them to experimental measurements. We find that an overall better agreement is achieved by the PBEsol functional, particularly for the high-lying optical phonon modes, as shown in Fig. 5(a). The better performance of PBEsol over PBE is also evident in the predicted $\kappa_{\mathrm{L}}$ : PBEsol predicts a higher value $\left[\kappa_{3,4 \mathrm{ph}}^{\mathrm{SCPH}}=2.3 \mathrm{~W} /(\mathrm{m} \cdot \mathrm{K})\right]$ than $\mathrm{PBE}\left[\kappa_{3,4 \mathrm{ph}}^{\mathrm{SCPH}}=1.8 \mathrm{~W} /(\mathrm{m} \cdot \mathrm{K})\right]$, and the former agrees better with the experimental range of $1.9-2.6 \mathrm{~W} /(\mathrm{m} \cdot \mathrm{K})$ $[83,84]$. Therefore, our following analysis and discussion will be based on the results obtained from the PBEsol functional. The energy cumulative $\kappa_{\mathrm{L}}$ in Fig. 5(b) shows that the majority of lattice heat conduction in $\mathrm{HgTe}$ is carried by acoustic phonons with frequencies less than $10 \mathrm{meV}$. In addition, acoustic phonons with frequencies less than $5 \mathrm{meV}$ contribute much more to $\kappa_{\mathrm{L}}$ than those with higher frequencies. The calculated $\kappa_{\mathrm{L}}$ considering only $3 \mathrm{ph}$ scattering has a value of $14.2 \mathrm{~W} /(\mathrm{m} \cdot \mathrm{K})$ at $300 \mathrm{~K}$ $[12.8 \mathrm{~W} /(\mathrm{m} \cdot \mathrm{K})$ without APRN, see Table I], consistent with an earlier study [147]. Remarkably, the 4ph scattering is able to significantly suppress the heat conduction contributed by those acoustic modes, reducing $\kappa_{\mathrm{L}}$ from $14.2 \mathrm{~W} /(\mathrm{m} \cdot \mathrm{K})$ to $2.3 \mathrm{~W} /(\mathrm{m} \cdot \mathrm{K})$ at $300 \mathrm{~K}$, a more than sixfold reduction. To the best of our knowledge, such a dramatic reduction due to 4 ph scattering is the largest one ever reported at room temperature, highlighting the unique behavior of anharmonic lattice dynamics in HgTe. The mean free path (MFP) cumulative $\kappa_{\mathrm{L}}$, as shown in Fig. 5(c), shows that $4 \mathrm{ph}$ scattering reduces the largest MFP down to $10^{3} \mathrm{~nm}$ and strongly suppresses heat transfer from thermal carriers with MFP larger than $50 \mathrm{~nm}$. As a rough estimation, creating nanostructures with sizes of about $100 \mathrm{~nm}$ is expected to further reduce $\kappa_{\mathrm{L}}$, but it would still be challenging to achieve a value of less than $1.0 \mathrm{~W} /(\mathrm{m} \cdot \mathrm{K})$.

We compare the $4 \mathrm{ph}$ scattering rates with the $3 \mathrm{ph}$ scattering rates in Fig. 5(d). Surprisingly, we see that $4 \mathrm{ph}$ scattering dominates over $3 \mathrm{ph}$ scattering for a large portion of phonon modes. In contrast to recent studies that show that acoustic modes are less affected by 4 ph scattering than optical modes [44,57], scattering rates of the acoustic modes in $\mathrm{HgTe}$ from 4ph interactions are much larger than those from 3ph interactions, especially for those acoustic modes with energy less than $5 \mathrm{meV}$ or between $7.5 \mathrm{meV}$ and $10 \mathrm{meV}$. Since these modes give major contributions to heat conduction in the 3 ph scattering picture, it then comes as no surprise that $\kappa_{\mathrm{L}}$ is heavily suppressed when 4 ph scattering is considered. To explore more details about the scattering processes, we decompose the total $4 \mathrm{ph}$ scattering rates into 
(a)


(b)



(e)

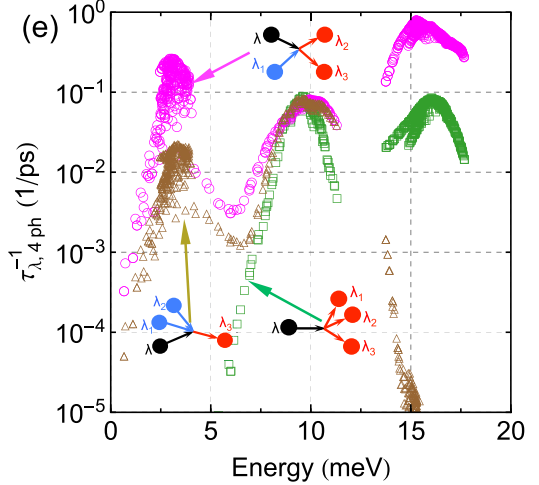

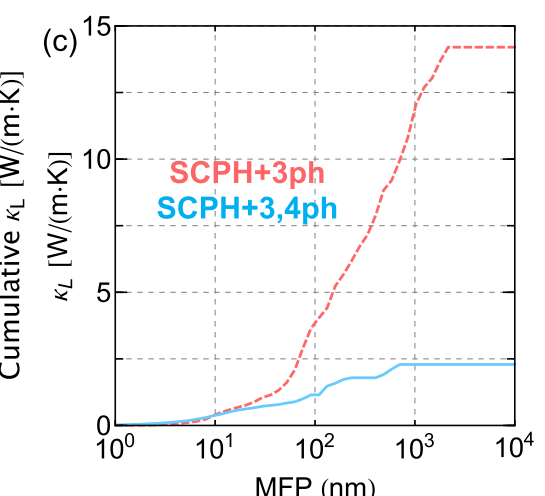

(f)



FIG. 5. Phonon dispersion, scattering rates, and lattice thermal conductivity of $\mathrm{HgTe}$. (a) Calculated phonon dispersions using the PBE functional (solid blue lines) and the PBEsol functional (solid orange lines) compared with experimental measurements (gray disks) $[148,149]$. (b) Cumulative (solid lines) and differential (dashed lines) $\kappa_{\mathrm{L}}$ calculated with (blue) and without (pink) 4ph scattering. (c) MFP cumulative $\kappa_{\mathrm{L}}$ with (blue) and without (pink) 4ph scattering. (d) Comparison of 3 ph scattering rates (empty red squares) and 4 ph scattering rates (empty blue circles). The solid black line indicates that the scattering rate equals phonon frequency. (e) Decomposed 4 ph scattering rates into the splitting $\left(\lambda \rightarrow \lambda_{1}+\lambda_{2}+\lambda_{3}\right)$, redistribution $\left(\lambda+\lambda_{1} \rightarrow \lambda_{2}+\lambda_{3}\right)$, and combination $\left(\lambda+\lambda_{1}+\lambda_{2} \rightarrow \lambda_{3}\right)$ processes, which are denoted as green circles, magneta squares, and brown triangles, respectively. (f) Calculated ratio of $\kappa_{3,4 \mathrm{ph}}^{\mathrm{SCPH}}$ to $\kappa_{3 \mathrm{ph}}^{\mathrm{SCPH}}$ as a function of the gap between acoustic and optical phonons (a-o gap). The insets show the phonon dispersions with longitudinal acoustic phonon branch manually scaled by three different constants, in accordance with the three values of a-o gap at the X point. All calculations are performed at $300 \mathrm{~K}$.

those coming from three individual processes, namely, splitting $\left(\lambda \rightarrow \lambda_{1}+\lambda_{2}+\lambda_{3}\right)$, redistribution $\left(\lambda+\lambda_{1} \rightarrow \lambda_{2}+\lambda_{3}\right)$, and combination $\left(\lambda+\lambda_{1}+\lambda_{2} \rightarrow \lambda_{3}\right)$ processes, respectively, as shown in Fig. 5(e). We find that the redistribution process dominates over the other two, which can be understood in terms of their associated scattering phase space. Considering the constraint enforced by the conservation of phonon energies in scattering processes, it is expected that combination processes are largely associated with phonon modes with low energies while the splitting processes are more relevant for high-lying phonon modes. The redistribution processes, which are more flexible in satisfying the energy conservation, are found to contribute many more $4 \mathrm{ph}$ scattering events, thus dominating both scattering phase space and scattering rates [54].

The dominant role of the $4 \mathrm{ph}$ scattering in HgTe suggests that the 3 ph scattering is relatively weaker. A similar picture has also been identified in BAs. As proposed in Ref. [30], the predicted very high $\kappa_{\mathrm{L}}$ based on $\mathrm{HA}+3 \mathrm{ph}$ in
BAs is attributable to the weak $3 \mathrm{ph}$ scattering of acoustic modes, resulting from (i) the large phonon band gap (a-o gap) between acoustic and optical modes, which effectively impedes acoustic modes scattering with optical modes, and (ii) the bunching of three acoustic branches, which suppresses the phase space for scattering among acoustic modes only. As a consequence, the $4 \mathrm{ph}$ scattering becomes important in BAs, being responsible for about a $40 \%$ reduction in $\kappa_{\mathrm{L}}$. It is intriguing to notice that the phonon dispersions of $\mathrm{HgTe}$ do not share these features. In contrast, HgTe has a much smaller a-o gap and a well-separated longitudinal acoustic (LA) phonon branch away from the transverse acoustic (TA) branches, which suggests new features that could potentially support relatively strong $4 \mathrm{ph}$ scattering. We have further examined the effects of the size of the a-o gap and the bunching of acoustic branches on $\kappa_{\mathrm{L}}$ in HgTe via manually scaling the energy of the LA branch as well as the group velocities with different constants. As shown in the inset of Fig. 5(f), increasing or decreasing the 
energy of the LA branch simultaneously decreases or increases both the a-o gap and the acoustic bunching. The resulting values of $\kappa_{3,4 \mathrm{ph}}^{\mathrm{SCPH}} / \kappa_{3 \mathrm{ph}}^{\mathrm{SCPH}}$ show that the largest relative reduction in $\kappa_{\mathrm{L}}$ due to $4 \mathrm{ph}$ scattering is not associated with the largest a-o gap and acoustic bunching. This observation indicates that $4 \mathrm{ph}$ scattering could be important even without a large a-o gap and acoustic bunching. The fact that the largest reduction associated with the unadjusted case, as shown in Fig. 5(f), reveals the nontrivial role of the shape of the LA branch in determining the strength of $4 \mathrm{ph}$ scattering relative to $3 \mathrm{ph}$ scattering, and therefore, an even smaller value of $\kappa_{3,4 \mathrm{ph}}^{\mathrm{SCPH}} / \kappa_{3 \mathrm{ph}}^{\mathrm{SCH}}$ may still exist.

\section{E. Nondiagonal contribution to lattice thermal conductivity beyond the phonon gas model}

The critical assumption adopted in our models is the quasiparticle nature of phonons; i.e., there exists a welldefined dispersion relation for phonons, and the broadening of phonon states is relatively weak. However, as pointed out by Allen and Feldman [109], the PBTE approach is accurate only when each phonon mode has either a long enough mean free path to define its wave vector or a lifetime long enough to define its frequency. It is thus expected that the PBTE approach only works well for weakly anharmonic systems with high $\kappa_{\mathrm{L}}$, but it fails for strongly anharmonic systems with low $\kappa_{\mathrm{L}}$. Moreover, strong broadening of phonon states would create an additional heat transport channel through the coupling of vibrational modes arising from off-diagonal terms in the heat-flux operator $[64,65]$, beyond the diagonal terms described by PGM/PBTE. Here, we examine the offdiagonal contribution to $\kappa_{\mathrm{L}}$ in these rocksalts and zinc blendes, which, when compared to the diagonal part, offers useful guidance on the validity of the phonon quasiparticle picture. The calculated ratio of the offdiagonal part $\kappa_{\mathrm{L}}^{\text {off-diagonal }}$ to the diagonal part $\kappa_{\mathrm{L}}^{\text {diagonal }}$ as a function of $\kappa_{\mathrm{L}}^{\text {diagonal }}$ is shown in Fig. 6(a). The rapidly decreasing trend confirms the minor contribution to the total $\kappa_{\mathrm{L}}$ from the off-diagonal part in compounds with high $\kappa_{\mathrm{L}}$, such as $\mathrm{BN} / \mathrm{P} / \mathrm{As}$. The off-diagonal part becomes increasingly important for low- $\kappa_{\mathrm{L}}$ compounds, and it contributes up to about $10 \%$ in $\mathrm{HgS} / \mathrm{Se} / \mathrm{Te}$. Specifically for $\mathrm{HgTe}$, the off-diagonal contribution increases $\kappa_{\mathrm{L}}$ from
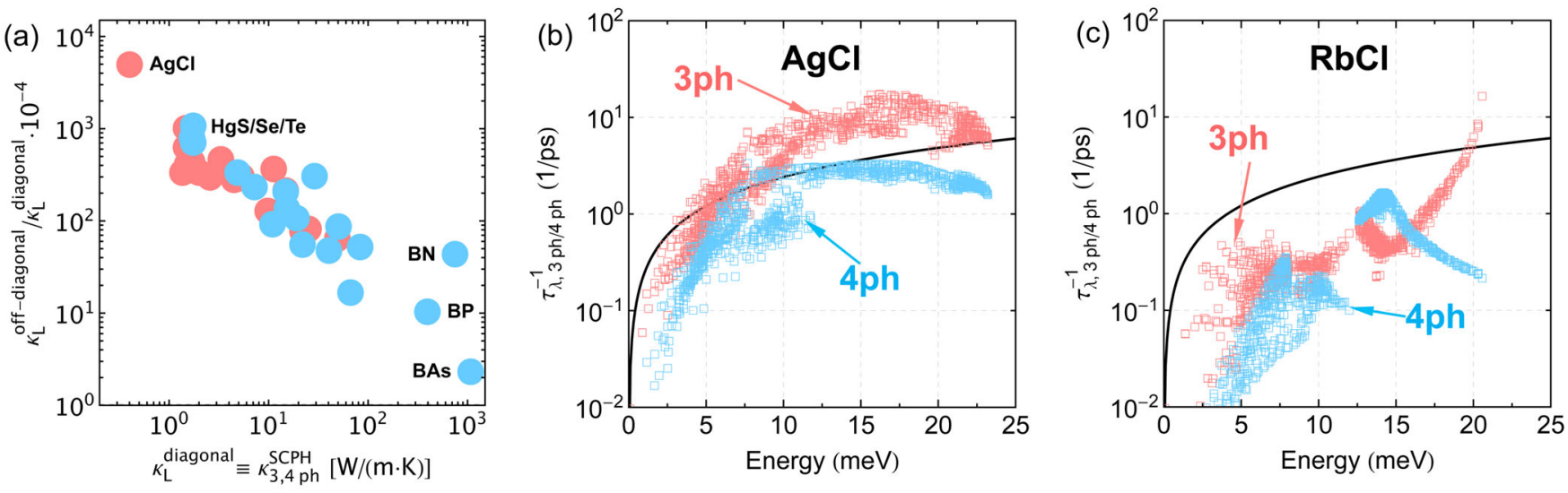

(d)



(e)

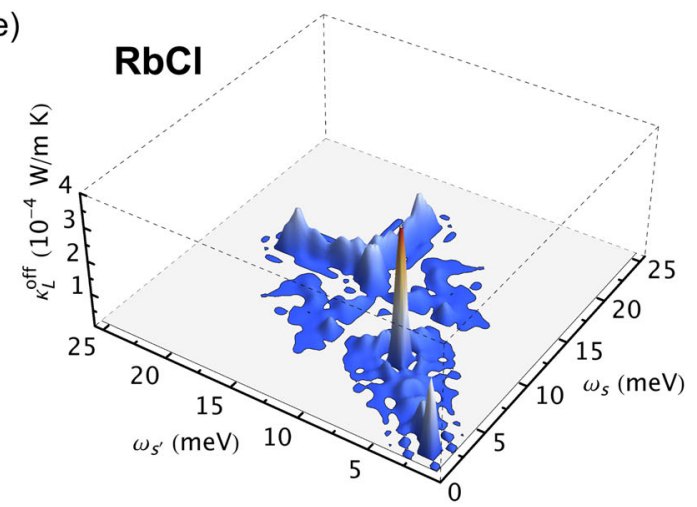

FIG. 6. (a) The ratio of lattice thermal conductivity from the off-diagonal terms $\left(\kappa_{\mathrm{L}}^{\text {off-diagonal }}\right)$ to the diagonal terms $\left(\kappa_{\mathrm{L}}^{\text {diagonal }}\right)$ in the heatflux operator as a function of $\kappa_{\mathrm{L}}^{\text {diagonal }}$ for selected rocksalts (red disks) and zinc blendes (blue disks). (b) Comparison of $3 \mathrm{ph}$ and $4 \mathrm{ph}$ scattering rates in $\mathrm{AgCl}$. (c) Same as panel (b), but for RbCl. The solid black lines in panels (b) and (c) denote the scattering rate with the same value of the phonon frequency. (d) Contour plots of the off-diagonal contribution to lattice thermal conductivity associated with various pairs of phonon frequencies $\left(\omega_{s}\right.$ and $\left.\omega_{s^{\prime}}\right)$ for $\mathrm{AgCl}$. (e) Same as panel (d), but for RbCl. All calculations are performed at $300 \mathrm{~K}$. 
$2.3 \mathrm{~W} /(\mathrm{m} \cdot \mathrm{K})$ to $2.4 \mathrm{~W} /(\mathrm{m} \cdot \mathrm{K})$ at $300 \mathrm{~K}$, making our theoretical prediction closer to the higher end of the experimental value of $2.6 \mathrm{~W} /(\mathrm{m} \cdot \mathrm{K})$. We also notice that this additional thermal transport channel may further mitigate the systematic underestimation of $\kappa_{\mathrm{L}}$ using the phonon gas model shown in Fig. 4(d), particularly for those compounds with low $\kappa_{\mathrm{L}}$ and strong phonon scattering.

Although the off-diagonal part is no longer negligible in low- $\kappa_{\mathrm{L}}$ systems, it is still much smaller than the diagonal part in almost all the selected binary compounds because enhancing the off-diagonal part requires significant broadening of phonon modes in order to promote wavelike tunneling and loss of coherence between vibrational eigenstates belonging to different branches. This condition is rather difficult to achieve in simple binary compounds due to their well-separated phonon branches. Nevertheless, a possible exception still exists, as indicated by a recent study [150] showing that the calculated $\kappa_{3 \mathrm{ph}}^{\mathrm{HA}}$ 's of rocksalt $\mathrm{AgCl}$ and $\mathrm{AgBr}$ have an ultralow value of about $0.2 \mathrm{~W} /(\mathrm{m} \cdot \mathrm{K})$ at $300 \mathrm{~K}$, only about one-fifth of the experimental value of $1.1 \mathrm{~W} /(\mathrm{m} \cdot \mathrm{K})$ [76]. These ultralow values of $\kappa_{\mathrm{L}}$ predicted based on PBTE suggest significant phonon broadening and the possible breakdown of the PGM in $\mathrm{AgCl}$ and $\mathrm{AgBr}$ at room temperature. To partially verify this proposal, we compute the diagonal and the off-diagonal components of $\kappa_{\mathrm{L}}$ in $\mathrm{AgCl}$ using the PBEsol functional. As shown in Fig. 6(a), the diagonal and the off-diagonal parts of $\kappa_{\mathrm{L}}$ in $\mathrm{AgCl}$ are of comparable importance, with the value of $\kappa_{\mathrm{L}}^{\text {off-diagonal }} / \kappa_{\mathrm{L}}^{\text {diagonal }}$ approaching 0.5 . Specifically, our results show that the off-diagonal contribution has a value of about $0.2 \mathrm{~W} /(\mathrm{m} \cdot \mathrm{K})$, leading to a total $\kappa_{\mathrm{L}}$ of $0.6 \mathrm{~W} /(\mathrm{m} \cdot \mathrm{K})$ at $300 \mathrm{~K}$, which brings the calculated $\kappa_{\mathrm{L}}$ closer to the experimental value. It is worth noting that despite the simple structure of $\mathrm{AgCl}$, the value of its offdiagonal contribution is comparable to that of $\mathrm{CsPbBr}_{3}$ $[0.3 \mathrm{~W} /(\mathrm{m} \cdot \mathrm{K})$ at $300 \mathrm{~K}]$, which has a complex structure containing 20 atoms per primitive cell [65].

As expected, the calculated phonon scattering rates in $\mathrm{AgCl}$ shown in Fig. 6(b) are exceptionally large for both $3 \mathrm{ph}$ and $4 \mathrm{ph}$ scattering. For a comparison, the phonon scattering rates in $\mathrm{RbCl}$ shown in Fig. 6(c) are, overall, much smaller, despite the fact that the two compounds have the same structure and similar formula mass. It is worth noting that the anharmonicity-induced phonon-phonon scattering is so strong in $\mathrm{AgCl}$ that most of the scattering rates are even larger than the frequencies of phonon modes themselves. This result provides concrete evidence for the possible breakdown of the phonon quasiparticle picture and the failure of PBTE for modeling $\kappa_{\mathrm{L}}$ in $\mathrm{AgCl}$. As a result, the significant broadening of phonon modes in $\mathrm{AgCl}$ induces wavelike tunneling among different phonon modes with the same wave vector, thus a large $\kappa_{\mathrm{L}}^{\text {off-diagonal }}$. Figures 6(d) and 6(e) show the off-diagonal coupling of vibrational eigenstates in $\mathrm{AgCl}$ and $\mathrm{RbCl}$, respectively. It can be seen that the strong phonon broadening in $\mathrm{AgCl}$ is able to couple phonon modes with large differences in frequencies, whereas in $\mathrm{RbCl}$, such coupling is much weaker and happens only among phonon modes with close energies.

\section{F. General remarks}

Before closing, we comment on the limitations of our theoretical framework and possible future directions. Despite being able to predict more accurate phonon frequencies and lifetimes by incorporating higher-order anharmonicity, we must bear in mind that our approach to compute $\kappa_{\mathrm{L}}$ still heavily relies on a fundamental proposal that those heat-carrying phonon states are well-behaved quasiparticles. Note that such an assumption applies to both the diagonal and the off-diagonal contributions to $\kappa_{\mathrm{L}}$ if they are formulated in terms of phonons. In reality, there are a plethora of scenarios that would lead to a partial or complete breakdown of the phonon quasiparticle picture, for instance, in the presence of strong disorder and anharmonicity $[109,151,152]$. Investigations into validating these fundamental assumptions will not only improve the accuracy of theoretical prediction but also provide insights into the microscopic mechanism of thermal transport, which may further guide experimentalists to favorably engineer heat transfer in functional materials. The large variations in the magnitude of $\kappa_{\mathrm{L}}$ and the strength of anharmonicity exhibited in the binary rocksalts and zinc blendes, as well as the structural simplicity, offer an excellent playground for the development, benchmarking, and improvement of theoretical models, which employ high-level theories beyond the state of the art. As a systematic way to lift the limitations imposed by the phonon quasiparticle picture, full frequency-dependent self-energies derived by the Green's function technique might be incorporated in modeling the phonon spectrum, as well as computing lattice thermal conductivity using the more general Kubo correlation-function formula $[17,153]$. Also, going beyond the perturbative evaluation of phonon self-energies might be necessary, as demonstrated by recent theoretical studies using stochastic approaches $[40,89,145]$.

Our results, which show the necessity of going beyond the three-phonon picture by including quartic anharmonicity, indicate that even higher-order anharmonicity might be important, especially in the presence of severe anharmonicity or at high temperatures. To overcome the limitation of anharmonic lattice dynamics calculations based on perturbation theory, one might resort to molecular dynamics simulations that naturally incorporate full anharmonicity. Though these molecular dynamics simulations can be expensive, the efficiency and accuracy might be significantly improved by taking advantage of high-quality empirical potentials including machine-learning potentials [154-157] and the modified embedded atom method (MEAM) potentials $[158,159]$. Another notable limitation for the current implementation of the Peierls-Boltzmann 
approach, as envisioned by Hardy [64] but rarely examined in practical calculations, is the lack of high-order terms of the energy flux, which may have important contributions to $\kappa_{\mathrm{L}}$ [160]. In addition, fully temperature-dependent thermal expansion calculated on top of the renormalized phonons might be included to simulate lattice dynamics and thermal transport properties, especially with an improved estimation of lattice parameters, for example, using the PBEsol functional $[142,161]$.

Concerning the application of the theoretical framework developed in this study, materials of particular interest might be those featuring rattling phonon modes, which have been demonstrated to display strong phonon hardening due to quartic anharmonicity at finite temperatures $[53,59,162]$. Another interesting class of compounds are perovskites, as they are known to exhibit phonon instabilities, and anharmonic phonon renormalization is indispensable for constructing physically sound phonon frequencies $[51,98,163]$. We expect future studies will be devoted to incorporating these advanced formalisms in first-principles simulations of anharmonic lattice dynamics and thermal transport properties of many other technically important materials.

\section{CONCLUSIONS}

In summary, we have systematically investigated the impact of quartic anharmonicity on room-temperature lattice dynamics and thermal transport properties of 19 binary rocksalt compounds $(\mathrm{MgO}, \mathrm{CaO}, \mathrm{SrO}, \mathrm{BaO}, \mathrm{LiH}$, LiF, NaF, NaCl, NaBr, NaI, KF, KCl, KBr, KI, RbCl, PbS, $\mathrm{PbSe}, \mathrm{PbTe}, \mathrm{AgCl}$ ) and 18 binary zinc blende compounds (AlAs, AlSb, BN, BP, BAs, CdSe, CdTe, GaP, GaSb, InP, InAs, InSb, ZnS, ZnSe, ZnTe, HgS, HgSe, HgTe). This is achieved by means of first-principles implementations of state-of-the-art thermal transport models based on the Peierls-Boltzmann approach, including anharmonic phonon renormalization and multiphonon scattering processes within a high-throughput phonon framework built on top of the OQMD.

We have performed detailed analyses of the lattice thermal conductivities calculated by progressively incorporating the effects of phonon frequency shifts and fourphonon scattering rates, which reveals strikingly distinctive effects of quartic anharmonicity on lattice thermal conductivity between binary rocksalts and zinc blendes. Specifically, strong anharmonic phonon renormalization due to quartic anharmonicity is observed in rocksalts, while in zinc blendes, it is rather weak, which in turn enhances the calculated lattice thermal conductivity for the former but leads to a negligible change in thermal conductivity for the latter. Four-phonon scattering rates, which are found to be generally important for most of the studied compounds, counteract the frequency shifts, resulting in an overall reduction in thermal conductivity of zinc blendes, but either enhanced or reduced thermal conductivity of rocksalts.
The calculated lattice thermal conductivities with full consideration of phonon frequency shifts and four-phonon scattering rates show noticeably improved agreement with experimental measurements with reduced deviation and variation. By correlating the lattice thermal conductivity and phonon scattering phase space, we have demonstrated that the importance of four-phonon scattering relative to the three-phonon scattering may be qualitatively assessed from the ratio of four-phonon to three-phonon scattering phase space, which is accessible from harmonic phonon dispersions.

We have identified strong four-phonon scattering in several compounds, including those already reported (e.g., BAs, $\mathrm{PbTe}, \mathrm{AlSb}, \mathrm{NaCl}$ ) and unreported (e.g., $\mathrm{NaF}$, InP, CdSe, ZnS), among which the $\mathrm{HgS} / \mathrm{Se} / \mathrm{Te}$ family exhibits unprecedented and up to about sixfold reduction in thermal conductivity due to four-phonon scattering. Detailed analysis of the phonon scattering rates in $\mathrm{HgTe}$ reveals the dominant role of the four-phonon scattering processes over the three-phonon scattering processes even in the acoustic region at room temperature. The case of $\mathrm{HgTe}$ unambiguously reveals that strong fourphonon scattering could be expected for compounds beyond those with large acoustic-optical phonon band gaps and bunching of acoustic modes.

We have examined the additional contribution to lattice thermal conductivity from off-diagonal terms in the heatflux operator by going beyond the phonon gas model, which only considers diagonal terms. It is found that the off-diagonal contribution is generally much smaller than the diagonal contribution when lattice thermal conductivity is high and the phonon quasiparticle picture is valid. For compounds with low lattice thermal conductivities, the offdiagonal contribution could comprise about $10 \%$ of the total lattice thermal conductivity (e.g., zinc blende $\mathrm{HgTe}$ ).

We have also identified an exceptional case, rocksalt $\mathrm{AgCl}$, which exhibits an anomalous off-diagonal contribution comparable to the diagonal contribution. This highly unusual behavior, especially when considering the rather simple crystal and well-separated phonon branches, arises from the intrinsically very strong anharmonic multiphonon scattering, which broadens phonon states and induces wavelike tunneling between different phonon states.

These findings-which highlight the importance of high-order phonon-phonon interactions, a route for predicting which compounds would benefit from four-phonon interactions based on harmonic calculations, and a possible breakdown of the conventional phonon gas modelprovide deep insights into the microscopic mechanism underlying the heat transport in simple crystalline compounds. The first-principles framework developed in this study, which embraces advanced lattice dynamics simulations, is universal, and the application of such a framework in large-scale phonon calculations in conjunction with machine-learning algorithms may provide valuable 
guidance for the rational design of new thermal management materials.

VASP (DFT calculations) is available at [164]; the ShengBTE code (thermal conductivity calculations based on three-phonon scattering) is available at [165]; CSLD (construction of higher-order force constants) is available at [166]. The custom codes (self-consistent phonon and fourphonon scattering calculations) in this work are available from the corresponding authors upon reasonable request.

\section{ACKNOWLEDGMENTS}

The authors acknowledge financial support received from (i) Toyota Research Institute (TRI) through the Accelerated Materials Design and Discovery program (development of high-throughput framework for lattice dynamics and thermal conductivity calculations), (ii) the Department of Energy, Office of Science, Basic Energy Sciences under Grant No. DE-SC0014520 (theory of anharmonic phonons), and (iii) the U.S. Department of Commerce and National Institute of Standards and Technology as part of the Center for Hierarchical Materials Design (CHiMaD) under Grant No. 70NANB14H012 (DFT calculations). This research used resources of the National Energy Research Scientific Computing Center, a DOE Office of Science User Facility supported by the Office of Science of the U.S. Department of Energy (U.S. Department of Energy Contract No. DEAC02-05CH11231) and the Extreme Science and Engineering Discovery Environment (National Science Foundation Contract No. ACI-1548562).

Note added.- Recently, we became aware of a related study by another group [104]. They investigated lattice thermal conductivity in 17 zinc blende compounds, including both three- and four-phonon interactions. Percentagewise, their reported reduction in lattice thermal conductivity due to four-phonon scattering is comparable to ours, although a different exchange correlation functional was used in their density functional theory calculations.

\section{APPENDIX A: SELF-CONSISTENT PHONON THEORY}

The anharmonic phonon renormalization of phonon frequency from quartic anharmonicity at finite temperatures is calculated using SCPH theory [90-93]. When formulated in the reciprocal space accounting for the firstorder correction from quartic anharmonicity in the diagonal form [51,87], the resulting SCPH equation reads

$$
\Omega_{\lambda}^{2}=\omega_{\lambda}^{2}+2 \Omega_{\lambda} \sum_{\lambda_{1}} I_{\lambda \lambda_{1}},
$$

where $\omega_{\lambda}$ is the bare frequency of phonon mode $\lambda$ calculated from the harmonic approximation and $\Omega_{\lambda}$ is the renormalized frequency including temperature effects. The quantity $I_{\lambda \lambda_{1}}$ is defined as

$$
I_{\lambda \lambda_{1}}=\frac{\hbar}{8 N} \frac{V^{(4)}\left(\lambda,-\lambda, \lambda_{1},-\lambda_{1}\right)}{\Omega_{\lambda} \Omega_{\lambda_{1}}}\left[1+2 n\left(\Omega_{\lambda_{1}}\right)\right],
$$

where $N, \hbar, n$, and $V^{(4)}\left(\lambda,-\lambda, \lambda_{1},-\lambda_{1}\right)$ are, respectively, the number of sampled wave vectors, the reduced Planck constant, phonon population, and the reciprocal representation of the fourth-order interatomic force constants (see detailed expression in the next section). The temperature effects in the formalism of SCPH are captured by the phonon population $n$ that obeys the Bose-Einstein statistics. Because of the mutual dependence between $\Omega_{\lambda}$ and $I_{\lambda \lambda_{1}}$, the SCPH equation (A1) can be solved in an iterative manner until a reasonable convergence is reached. It is worth mentioning that $I_{\lambda \lambda_{1}}$ can be interpreted as the contribution from mode $\lambda_{1}$ to the anharmonic renormalization of $\lambda$. When $\lambda_{1}$ and $\lambda$ indicate the same phonon mode, SCPH can be viewed as the renormalization performed in the frozen phonon picture of mode $\lambda$, including a quartic contribution to the potential energy surface. We note that, in this study, we do not consider the cubic-anharmonicity-induced frequency shift, which is a second-order effect and has full frequency dependence. In addition, anharmonic phonon renormalization is performed using the DFT fully relaxed structure without considering thermal expansion, unless stated otherwise. It is worth noting that a more advanced SCPH scheme, including off-diagonal terms that enable updating phonon eigenvectors (polarization mixing), has recently been developed, which is implemented in the publicly available ALAMODE package [51,167].

\section{APPENDIX B: PHONON SCATTERING RATES}

The key ingredients entering the phonon Boltzmann transport equation are the phonon scattering rates arising from both multiphonon interactions and phonon-isotope scattering. For the intrinsic phonon-phonon scattering, we consider $3 \mathrm{ph}$ and $4 \mathrm{ph}$ scattering processes. The phonon self-energy arising from both $3 \mathrm{ph}$ and $4 \mathrm{ph}$ interactions has been derived by many authors [10,47-50,153] using Green's function technique. Recently, Feng and Ruan have derived $3 \mathrm{ph}$ and $4 \mathrm{ph}$ scattering rates by treating the cubic and quartic anharmonic terms as perturbations using Fermi's golden rule of time-dependent perturbation theory [52]. In this study, we adopt their formulas for both $3 \mathrm{ph}$ and 4 ph scattering rates, which, under SMRTA, read

$$
\tau_{3 \mathrm{ph}, \lambda}^{-1}=\sum_{\lambda_{1} \lambda_{2}}\left\{\frac{1}{2}\left(1+n_{\lambda_{1}}+n_{\lambda_{2}}\right) \zeta_{-}+\left(n_{\lambda_{1}}-n_{\lambda_{2}}\right) \zeta_{+}\right\},
$$




$$
\begin{aligned}
\tau_{\mathrm{pph}, \lambda}^{-1}= & \sum_{\lambda_{1} \lambda_{2} \lambda_{3}}\left\{\frac{n_{\lambda_{1}} n_{\lambda_{2}} n_{\lambda_{3}}}{6 n_{\lambda}} \zeta_{--}+\frac{\left(1+n_{\lambda_{1}}\right) n_{\lambda_{2}} n_{\lambda_{3}}}{2 n_{\lambda}} \zeta_{+-}\right. \\
& \left.+\frac{\left(1+n_{\lambda_{1}}\right)\left(1+n_{\lambda_{2}}\right) n_{\lambda_{3}}}{2 n_{\lambda}} \zeta_{++}\right\},
\end{aligned}
$$

where $\lambda$ is a composite index for a phonon mode with wave vector $\mathbf{q}$ and branch $s$, and $n$ is the phonon population following Bose-Einstein statistics, with

$$
\zeta_{ \pm}=\frac{\pi \hbar}{4 N}\left|V^{(3)}\left(\lambda, \pm \lambda_{1},-\lambda_{2}\right)\right|^{2} \pm \frac{\delta\left(\omega_{\lambda} \pm \omega_{\lambda_{1}}-\omega_{\lambda_{2}}\right)}{\omega_{\lambda} \omega_{\lambda_{1}} \omega_{\lambda_{2}}}
$$

and

$$
\begin{aligned}
\zeta_{ \pm \pm}= & \frac{\pi \hbar^{2}}{8 N^{2}}\left|V^{(4)}\left(\lambda, \pm \lambda_{1}, \pm \lambda_{2},-\lambda_{3}\right)\right|^{2} \\
& \times \Delta_{ \pm \pm} \frac{\delta\left(\omega_{\lambda} \pm \omega_{\lambda_{1}} \pm \omega_{\lambda_{2}}-\omega_{\lambda_{3}}\right)}{\omega_{\lambda} \omega_{\lambda_{1}} \omega_{\lambda_{2}} \omega_{\lambda_{3}}}
\end{aligned}
$$

where $V^{(3)}\left(\lambda, \pm \lambda_{1},-\lambda_{2}\right)$ and $V^{(4)}\left(\lambda, \pm \lambda_{1}, \pm \lambda_{2},-\lambda_{3}\right)$ are

$$
\begin{aligned}
V^{(3)}\left(\lambda, \pm \lambda_{1},-\lambda_{2}\right)= & \sum_{b, l_{1} b_{1}, l_{2} b_{2}} \sum_{\alpha \alpha_{1} \alpha_{2}} \Phi_{0 b, l_{1} b_{1}, l_{2} b_{2}}^{\alpha \alpha_{1} \alpha_{2}} \\
& \times \frac{e_{\alpha b}^{\lambda} e_{\alpha_{1} b_{1}}^{ \pm \lambda_{1}} e_{\alpha_{2} b_{2}}^{-\lambda_{2}}}{\sqrt{m_{b} m_{b_{1}} m_{b_{2}}}} e^{ \pm i \mathbf{q}_{1} \cdot \mathbf{r}_{1}-i \mathbf{q}_{2} \cdot \mathbf{r}_{2}}
\end{aligned}
$$

and

$$
\begin{aligned}
V^{(4)} & \left(\lambda, \pm \lambda_{1}, \pm \lambda_{2},-\lambda_{3}\right) \\
= & \sum_{b, l_{1} b_{1}, l_{2} b_{2}, l_{3} b_{3}} \sum_{\alpha \alpha_{1} \alpha_{2} \alpha_{3}} \Phi_{0 b, l_{1} b_{1}, l_{2} b_{2}, l_{3} b_{3}}^{\alpha \alpha_{1} \alpha_{2} \alpha_{3}} \\
& \times \frac{e_{\alpha b}^{\lambda} e_{\alpha_{1} b_{1}}^{ \pm \lambda_{1}} e_{\alpha_{2} b_{2}}^{ \pm \lambda_{2}} e_{\alpha_{3} b_{3}}^{-\lambda_{3}}}{\sqrt{m_{b} m_{b_{1}} m_{b_{2}} m_{b_{3}}}} e^{ \pm i \mathbf{q}_{1} \cdot \mathbf{r}_{1} \pm i \mathbf{q}_{2} \cdot \mathbf{r}_{l_{2}}-i \mathbf{q}_{3} \cdot \mathbf{r}_{l_{3}}},
\end{aligned}
$$

where $l, b$, and $\alpha$ index the primitive cell, basis atom, and Cartesian coordinate, respectively. Atomic mass and the lattice vector of primitive cells are denoted, respectively, by $m$ and $\mathbf{r}$. The phonon frequency and eigenvector are denoted, respectively, by $\omega_{\lambda}$ and $e^{\lambda}$, and $\Phi_{0 b, l_{1} b_{1}, l_{2} b_{2}}^{\alpha \alpha_{1} \alpha_{2}}$ and $\Phi_{0 b, l_{1} b_{1}, l_{2} b_{2}, l_{3} b_{3}}^{\alpha \alpha_{1} \alpha_{2} \alpha_{3}}$ are the third- and fourth-order interatomic force constants (IFCs), respectively. For both three- and four-phonon scattering processes, the momentum conservation is strictly enforced by $\delta$ functions, namely, $\Delta_{ \pm} \equiv$ $\delta\left(\mathbf{q} \pm \mathbf{q}_{1}-\mathbf{q}_{2}\right)$ and $\Delta_{ \pm \pm} \equiv \delta\left(\mathbf{q} \pm \mathbf{q}_{1} \pm \mathbf{q}_{2}-\mathbf{q}_{3}\right) ;$ thus, energy conservation is approximated by adaptive $[114,168]$ and regular Gaussian smearing in evaluating $\tau_{3 \mathrm{ph}, \lambda}^{-1}$ and $\tau_{4 \mathrm{ph}, \lambda}^{-1}$, respectively. The $3 \mathrm{ph}$ and $4 \mathrm{ph}$ scattering phases used in the main text are defined as $\Theta_{3 \mathrm{ph}}=$ $\sum_{\lambda} \Theta_{3 \mathrm{ph}, \lambda}$ and $\Theta_{4 \mathrm{ph}}=\sum_{\lambda} \Theta_{4 \mathrm{ph}, \lambda}$, respectively, wherein the evaluation of $\Theta_{3 \mathrm{ph}, \lambda}$ and $\Theta_{4 \mathrm{ph}, \lambda}$ resembles that of $\tau_{3 \mathrm{ph}, \lambda}^{-1}$ and $\tau_{4 \mathrm{ph}, \lambda}^{-1}$ except that $\zeta_{ \pm}$and $\zeta_{ \pm \pm}$are replaced with

$$
\zeta_{ \pm}^{\prime}=\Delta_{ \pm} \frac{\delta\left(\omega_{\lambda} \pm \omega_{\lambda_{1}}-\omega_{\lambda_{2}}\right)}{\omega_{\lambda} \omega_{\lambda_{1}} \omega_{\lambda_{2}}}
$$

and

$$
\zeta_{ \pm \pm}^{\prime}=\Delta_{ \pm \pm} \frac{\delta\left(\omega_{\lambda} \pm \omega_{\lambda_{1}} \pm \omega_{\lambda_{2}}-\omega_{\lambda_{3}}\right)}{\omega_{\lambda} \omega_{\lambda_{1}} \omega_{\lambda_{2}} \omega_{\lambda_{3}}}
$$

respectively.

Additional phonon scattering also comes from naturally occurring isotopes [95-97], denoted as $\tau_{\text {isotope }, \lambda}^{-1}$, which has been implemented in the ShengBTE package [114] and reads

$$
\tau_{\text {isotope }, \lambda}^{-1}=\frac{\pi \omega_{\lambda}^{2}}{2 N} \sum_{b} g_{b}\left|\mathbf{e}_{b}^{\lambda *} \cdot \mathbf{e}_{b}^{\lambda_{1}}\right|^{2} \delta\left(\omega_{\lambda}-\omega_{\lambda_{1}}\right),
$$

where $g_{b}=\sum_{i} x_{b}^{i}\left(1-m_{b}^{i} / \bar{m}_{b}\right)^{2}$ is the Pearson deviation coefficient of the masses $m_{b}^{i}$ of isotopes $i$ of atom $b$ found with probability $0<x_{b}^{i} \leq 1$ and $\bar{m}_{b}=\sum_{i} x_{b}^{i} m_{b}^{i}$.

\section{APPENDIX C: INTERATOMIC FORCE CONSTANTS}

The key elements entering the SCPH and phonon scattering rates are the IFCs. In this study, we used CSLD [61-63] to extract both harmonic and anharmonic IFCs. In brief, CSLD utilizes the compressive sensing technique $[125,169]$ to select the physically important IFCs from the force-displacement data generated from displaced atomic configurations under the constraints enforced by space group symmetry. It has been demonstrated that CSLD is particularly powerful for exacting high-order IFCs and requires much less supercell calculations than the regular small-displacement method wherein only a few atoms relevant to a specific IFC are displaced [114,170]. A specific example has been given for clathrates compounds with complex structures, and it shows that only 20 supercell structures are required for CSLD to extract third-order IFCs that lead to lattice thermal conductivity comparable to those from the small-displacement method, which requires more than 500 supercells [62]. We refer readers to Ref. [62] for more details. It is worth mentioning that the reliability of CSLD has been examined and confirmed in many studies [51,53,171-173]. In this work, we adopt convergence criteria for CSLD-extracted IFCs following our recent work on PbTe [45] and GeTe [54], which has been carefully examined and will be detailed below for both rocksalt and zinc blende compounds. First, we construct $4 \times 4 \times 4$ supercells from the fully relaxed supercell and generate random small displacements in the range from $0.01 \AA$ to $0.04 \AA$. Then, the cumulative harmonic IFCs were 
constructed by CSLD considering all pair interactions within the supercell. Second, we generate physically relevant atomic displacements at $300 \mathrm{~K}$ using the quantum covariance matrix of atomic displacement [45] calculated from the harmonic IFCs and extract the third-order and fourth-order IFCs using the force-displacement data that exclude the harmonic contribution. Note that these atomic displacements are usually much larger and system dependent, which, in principle, capture the strength of highorder IFCs better. The cutoff distances for the third- and fourth-order IFCs are limited up to the fifth and second nearest neighbors, respectively, in line with our convergence test for $\mathrm{PbTe} / \mathrm{GeTe}[45,54]$ and other studies [57]. Owing to the advanced algorithm implemented by CSLD and the highly symmetric structure of binary rocksalts and zinc blendes, usage of $304 \times 4 \times 4$ supercells for each compound already achieves good convergence, and the resulting lattice thermal conductivity mostly varies within about $5 \%$ compared to those obtained using significantly more supercells.

[1] L. E. Bell, Cooling, Heating, Generating Power, and Recovering Waste Heat with Thermoelectric Systems, Science 321, 1457 (2008).

[2] A. Mills, Heat and Mass Transfer, Irwin Graphics Series (Taylor \& Francis, London, 1995).

[3] S. L. Shinde and J. Goela, High Thermal Conductivity Materials (Springer, New York, 2006).

[4] R. R. Heikes and R. W. Ure, Thermoelectricity: Science and Engineering (Interscience, New York, 1961).

[5] D. Khokhlov, Lead Chalcogenides: Physics and Applications, Optoelectronic Properties of Semiconductors and Superlattices (Taylor \& Francis, London, 2002).

[6] J. S. Kang, M. Li, H. Wu, H. Nguyen, and Y. Hu, Experimental Observation of High Thermal Conductivity in Boron Arsenide, Science 361, 575 (2018).

[7] S. Li, Q. Zheng, Y. Lv, X. Liu, X. Wang, P. Y. Huang, D. G. Cahill, and B. Lv, High Thermal Conductivity in Cubic Boron Arsenide Crystals, Science 361, 579 (2018).

[8] F. Tian et al., Unusual High Thermal Conductivity in Boron Arsenide Bulk Crystals, Science 361, 582 (2018).

[9] M. Born and K. Huang, Dynamical Theory of Crystal Lattices, International Series of Monographs on Physics (Clarendon, Oxford, 1988).

[10] A. A. Maradudin and A. E. Fein, Scattering of Neutrons by an Anharmonic Crystal, Phys. Rev. 128, 2589 (1962).

[11] R. A. Cowley, Anharmonic Crystals, Rep. Prog. Phys. 31, 123 (1968).

[12] G. P. Srivastava, The Physics of Phonons (Taylor \& Francis, London, 1990).

[13] D. C. Wallace, Thermodynamics of Crystals, Dover Books on Physics (Dover, New York, 1998).

[14] P. Hohenberg and W. Kohn, Inhomogeneous Electron Gas, Phys. Rev. 136, B864 (1964).
[15] W. Kohn and L.J. Sham, Self-Consistent Equations Including Exchange and Correlation Effects, Phys. Rev. 140, A1133 (1965).

[16] M. Toda, R. Kubo, R. Kubo, M. Toda, N. Saito, N. Hashitsume, and N. Hashitsume, Statistical Physics II: Nonequilibrium Statistical Mechanics, Springer Series in Solid-State Sciences (Springer, Berlin, Heidelberg, 2012).

[17] G. D. Mahan, Many-Particle Physics, Physics of Solids and Liquids (Springer, New York, 2000).

[18] J.-S. Wang, Quantum Thermal Transport from Classical Molecular Dynamics, Phys. Rev. Lett. 99, 160601 (2007).

[19] J. E. Turney, E. S. Landry, A. J. H. McGaughey, and C. H. Amon, Predicting Phonon Properties and Thermal Conductivity from Anharmonic Lattice Dynamics Calculations and Molecular Dynamics Simulations, Phys. Rev. B 79, 064301 (2009).

[20] N. Shulumba, O. Hellman, and A. J. Minnich, Lattice Thermal Conductivity of Polyethylene Molecular Crystals from First-Principles Including Nuclear Quantum Effects, Phys. Rev. Lett. 119, 185901 (2017).

[21] R. E. Peierls, Quantum Theory of Solids, International Series of Monographs on Physics (Clarendon, Oxford, 1996).

[22] D. A. Broido, M. Malorny, G. Birner, N. Mingo, and D. A. Stewart, Intrinsic Lattice Thermal Conductivity of Semiconductors from First Principles, Appl. Phys. Lett. 91, 231922 (2007).

[23] J. Garg, N. Bonini, B. Kozinsky, and N. Marzari, Role of Disorder and Anharmonicity in the Thermal Conductivity of Silicon-Germanium Alloys: A First-Principles Study, Phys. Rev. Lett. 106, 045901 (2011).

[24] K. Esfarjani, G. Chen, and H. T. Stokes, Heat Transport in Silicon from First-Principles Calculations, Phys. Rev. B 84, 085204 (2011).

[25] L. Chaput, Direct Solution to the Linearized Phonon Boltzmann Equation, Phys. Rev. Lett. 110, 265506 (2013).

[26] G. Fugallo, M. Lazzeri, L. Paulatto, and F. Mauri, Ab Initio Variational Approach for Evaluating Lattice Thermal Conductivity, Phys. Rev. B 88, 045430 (2013).

[27] A. Cepellotti and N. Marzari, Thermal Transport in Crystals as a Kinetic Theory of Relaxons, Phys. Rev. X 6, 041013 (2016).

[28] J. S. Reid, Phonon Gas, Phys. Educ. 11, 348 (1976).

[29] A. Ward, D. A. Broido, D. A. Stewart, and G. Deinzer, Ab initio, Phys. Rev. B 80, 125203 (2009).

[30] L. Lindsay, D. A. Broido, and T. L. Reinecke, FirstPrinciples Determination of Ultrahigh Thermal Conductivity of Boron Arsenide: A Competitor for Diamond?, Phys. Rev. Lett. 111, 025901 (2013).

[31] A. Cepellotti, G. Fugallo, L. Paulatto, M. Lazzeri, F. Mauri, and N. Marzari, Phonon Hydrodynamics in TwoDimensional Materials, Nat. Commun. 6, 6400 (2015).

[32] Z. Tian, J. Garg, K. Esfarjani, T. Shiga, J. Shiomi, and G. Chen, Phonon Conduction in PbSe, PbTe, and $\mathrm{PbTe}_{1-x} \mathrm{Se}_{x}$ from First-Principles Calculations, Phys. Rev. B 85, 184303 (2012).

[33] J. Shiomi, K. Esfarjani, and G. Chen, Thermal Conductivity of Half-Heusler Compounds from First-Principles Calculations, Phys. Rev. B 84, 104302 (2011). 
[34] W. Li and N. Mingo, Thermal Conductivity of Fully Filled Skutterudites: Role of the Filler, Phys. Rev. B 89, 184304 (2014).

[35] W. Li and N. Mingo, Ultralow Lattice Thermal Conductivity of the Fully Filled Skutterudite $\mathrm{YbFe}_{4} \mathrm{Sb}_{12}$ Due to the Flat Avoided-Crossing Filler Modes, Phys. Rev. B 91, 144304 (2015).

[36] A. H. Romero, E. K. U. Gross, M. J. Verstraete, and O. Hellman, Thermal Conductivity in PbTe from First Principles, Phys. Rev. B 91, 214310 (2015).

[37] T. Tadano, Y. Gohda, and S. Tsuneyuki, Impact of Rattlers on Thermal Conductivity of a Thermoelectric Clathrate: A First-Principles Study, Phys. Rev. Lett. 114, 095501 (2015).

[38] J. Carrete, N. Mingo, and S. Curtarolo, Low Thermal Conductivity and Triaxial Phononic Anisotropy of SnSe, Appl. Phys. Lett. 105, 101907 (2014).

[39] J. M. Skelton, S. C. Parker, A. Togo, I. Tanaka, and A. Walsh, Thermal Physics of the Lead Chalcogenides PbS, PbSe, and PbTe from First Principles, Phys. Rev. B 89, 205203 (2014).

[40] U. Aseginolaza, R. Bianco, L. Monacelli, L. Paulatto, M. Calandra, F. Mauri, A. Bergara, and I. Errea, Phonon Collapse and Second-Order Phase Transition in Thermoelectric SnSe, Phys. Rev. Lett. 122, 075901 (2019).

[41] B. Lv, Y. Lan, X. Wang, Q. Zhang, Y. Hu, A. J. Jacobson, D. Broido, G. Chen, Z. Ren, and C.-W. Chu, Experimental Study of the Proposed Super-Thermal-Conductor: BAs, Appl. Phys. Lett. 106, 074105 (2015).

[42] J. Kim, D. A. Evans, D. P. Sellan, O. M. Williams, E. Ou, A. H. Cowley, and L. Shi, Thermal and Thermoelectric Transport Measurements of an Individual Boron Arsenide Microstructure, Appl. Phys. Lett. 108, 201905 (2016).

[43] Q. Zheng, C. A. Polanco, M.-H. Du, L. R. Lindsay, M. Chi, J. Yan, and B.C. Sales, Antisite Pairs Suppress the Thermal Conductivity of BAs, Phys. Rev. Lett. 121, 105901 (2018).

[44] T. Feng, L. Lindsay, and X. Ruan, Four-Phonon Scattering Significantly Reduces Intrinsic Thermal Conductivity of Solids, Phys. Rev. B 96, 161201 (2017).

[45] Y. Xia, Revisiting Lattice Thermal Transport in PbTe: The Crucial Role of Quartic Anharmonicity, Appl. Phys. Lett. 113, 073901 (2018).

[46] N. K. Ravichandran and D. Broido, Unified FirstPrinciples Theory of Thermal Properties of Insulators, Phys. Rev. B 98, 085205 (2018).

[47] D. W. Jepsen and R. F. Wallis, Effect of Quartic Anharmonicity on the Infrared Absorption of Alkali Halide Crystals, Phys. Rev. 125, 1496 (1962).

[48] I. P. Ipatova, A. A. Maradudin, and R. F. Wallis, Temperature Dependence of the Width of the Fundamental Lattice-Vibration Absorption Peak in Ionic Crystals. II. Approximate Numerical Results, Phys. Rev. 155, 882 (1967).

[49] R. S. Tripathi and K. N. Pathak, Self-Energy of Phonons in an Anharmonic Crystal to O(4), Nuovo Cimento Soc. Ital. Fis. B 21, 289 (1974).

[50] M. Balkanski, R. F. Wallis, and E. Haro, Anharmonic Effects in Light Scattering Due to Optical Phonons in Silicon, Phys. Rev. B 28, 1928 (1983).
[51] T. Tadano and S. Tsuneyuki, Self-Consistent Phonon Calculations of Lattice Dynamical Properties in Cubic $\mathrm{SrTiO}_{3}$ with First-Principles Anharmonic Force Constants, Phys. Rev. B 92, 054301 (2015).

[52] T. Feng and X. Ruan, Quantum Mechanical Prediction of Four-Phonon Scattering Rates and Reduced Thermal Conductivity of Solids, Phys. Rev. B 93, 045202 (2016).

[53] T. Tadano and S. Tsuneyuki, Quartic Anharmonicity of Rattlers and Its Effect on Lattice Thermal Conductivity of Clathrates from First Principles, Phys. Rev. Lett. 120, 105901 (2018).

[54] Y. Xia and M. K. Y. Chan, Anharmonic Stabilization and Lattice Heat Transport in Rocksalt-GeTe, Appl. Phys. Lett. 113, 193902 (2018).

[55] T. Feng and X. Ruan, Four-Phonon Scattering Reduces Intrinsic Thermal Conductivity of Graphene and the Contributions from Flexural Phonons, Phys. Rev. B 97, 045202 (2018).

[56] X. Gu, Z. Fan, H. Bao, and C. Y. Zhao, Revisiting PhononPhonon Scattering in Single-Layer Graphene, Phys. Rev. B 100, 064306 (2019).

[57] X. Yang, T. Feng, J. Li, and X. Ruan, Stronger Role of Four-Phonon Scattering Than Three-Phonon Scattering in Thermal Conductivity of III-V Semiconductors at Room Temperature, Phys. Rev. B 100, 245203 (2019).

[58] M. Puligheddu, Y. Xia, M. Chan, and G. Galli, Computational Prediction of Lattice Thermal Conductivity: A Comparison of Molecular Dynamics and Boltzmann Transport Approaches, Phys. Rev. Mater. 3, 085401 (2019).

[59] Y. Xia and V. Ozoliņš, Impact of Temperature-Dependent Rattling Phonons on Lattice Thermal Transport in $\mathrm{Ag}_{6} \mathrm{Ge}_{10} \mathrm{P}_{12}$, arXiv:1903.08800.

[60] Y. Xia, V. Hegde, K. Pal, D. Gaines, J. He, M. Aykol, and C. Wolverton, High-Throughput Finite-Temperature Materials Properties Including Higher-Order Anharmonicity (unpublished).

[61] F. Zhou, W. Nielson, Y. Xia, and V. Ozoliņš, Lattice Anharmonicity and Thermal Conductivity from Compressive Sensing of First-Principles Calculations, Phys. Rev. Lett. 113, 185501 (2014).

[62] F. Zhou, W. Nielson, Y. Xia, and V. Ozoliņš, Compressive Sensing Lattice Dynamics. I. General Formalism, Phys. Rev. B 100, 184308 (2019).

[63] F. Zhou, B. Sadigh, D. Åberg, Y. Xia, and V. Ozoliṇš, Compressive Sensing Lattice Dynamics. II. Efficient Phonon Calculations and Long-Range Interactions, Phys. Rev. B 100, 184309 (2019).

[64] R. J. Hardy, Energy-Flux Operator for a Lattice, Phys. Rev. 132, 168 (1963).

[65] M. Simoncelli, N. Marzari, and F. Mauri, Unified Theory of Thermal Transport in Crystals and Glasses, Nat. Phys. 15, 809 (2019).

[66] II-VI and I-VII Compounds: Semimagnetic Compounds, in Landolt-Bornstein-Group III Condensed Matter, edited by O. Madelung, U. Rossler, and M. Schulz (SpringerVerlag, Berlin, Heidelberg, 1999), Vol. 41B.

[67] G. A. Slack, Nonmetallic Crystals with High Thermal Conductivity, J. Phys. Chem. Solids 34, 321 (1973). 
[68] S. Andersson and G. Backstrom, Thermal Conductivity and Heat Capacity of Single-Crystal LiF and $\mathrm{CaF}_{2}$ under Hydrostatic Pressure, J. Phys. C 20, 5951 (1987).

[69] B. Håkansson and R. G. Ross, Thermal Conductivity of Solid NaF under High Pressure, Int. J. Thermophys. 6, 353 (1985).

[70] B. Håkansson and P. Andersson, Thermal Conductivity and Heat Capacity of Solid $\mathrm{NaCl}$ and NaI under Pressure, J. Phys. Chem. Solids 47, 355 (1986).

[71] I. Sigalas, B. Håkanson, and P. Andersson, Thermal Conductivity and Heat Capacity of Solid $\mathrm{NaBr}$ under Pressure, Int. J. Thermophys. 6, 177 (1985).

[72] R. J. Gummow and I. Sigalas, The Thermal Conductivity and Thermal Diffusivity of KF as a Function of Pressure between 0 and 3.8 GPa, J. Phys. C 20, L61 (1987).

[73] S. Pettersson, The Minimum Thermal Conductivity of Alkali Halides, J. Phys. Condens. Matter 1, 361 (1989).

[74] A. A. El-Sharkawy, A. M. Abou El-Azm, M. I. Kenawy, A. S. Hillal, and H. M. Abu-Basha, Thermophysical Properties of Polycrystalline $\mathrm{PbS}, \mathrm{PbSe}$, and PbTe in the Temperature Range 300-700 K, Int. J. Thermophys. 4, 261 (1983).

[75] G. A. Akhmedova and D. S. Abdinov, Effect of Thallium Doping on the Thermal Conductivity of PbTe Single Crystals, Inorg. Mater. 45, 854 (2009).

[76] R. G. Ross, P. Andersson, and G. Bäckström, Thermal Conductivity and Heat Capacity of Solid $\mathrm{AgCl}$ under Pressure, Int. J. Thermophys. 2, 289 (1981).

[77] M. A. Afromowitz, Thermal Conductivity of $\mathrm{Ga}_{1 x} \mathrm{Al}_{x}$ As Alloys, J. Appl. Phys. 44, 1292 (1973).

[78] E. F. Steigmeier and I. Kudman, Thermal Conductivity of III-V Compounds at High Temperatures, Phys. Rev. 132, 508 (1963).

[79] E. F. Steigmeier and I. Kudman, Acoustical-Optical Phonon Scattering in Ge, Si, and III-V Compounds, Phys. Rev. 141, 767 (1966).

[80] Q. Zheng, Sh. Li, C. Li, Y. Lv, X. Liu, P. Y. Huang, D. A. Broido, B. Lv, and D. G. Cahill, High Thermal Conductivity in Isotopically Enriched Cubic Boron Phosphide, Adv. Funct. Mater. 28, 1805116 (2018).

[81] Y. Kumashiro, T. Mitsuhashi, S. Okaya, F. Muta, T. Koshiro, Y. Takahashi, and M. Mirabayashi, Thermal Conductivity of a Boron Phosphide Single Crystal Wafer up to High Temperature, J. Appl. Phys. 65, 2147 (1989).

[82] J. S. Kang, H. Wu, and Y. Hu, Thermal Properties and Phonon Spectral Characterization of Synthetic Boron Phosphide for High Thermal Conductivity Applications, Nano Lett. 17, 7507 (2017).

[83] G. A. Slack, Thermal Conductivity of II-VI Compounds and Phonon Scattering by $\mathrm{Fe}^{2+}$ Impurities, Phys. Rev. B 6 , 3791 (1972).

[84] C. R. Whitsett and D. A. Nelson, Lattice Thermal Conductivity of p-Type Mercury Telluride, Phys. Rev. B 5, 3125 (1972).

[85] A. Debernardi, S. Baroni, and E. Molinari, Anharmonic Phonon Lifetimes in Semiconductors from DensityFunctional Perturbation Theory, Phys. Rev. Lett. 75, 1819 (1995).

[86] P. Souvatzis, O. Eriksson, M. I. Katsnelson, and S. P. Rudin, Entropy Driven Stabilization of Energetically
Unstable Crystal Structures Explained from First Principles Theory, Phys. Rev. Lett. 100, 095901 (2008).

[87] I. Errea, Bruno Rousseau, and Aitor Bergara, Anharmonic Stabilization of the High-Pressure Simple Cubic Phase of Calcium, Phys. Rev. Lett. 106, 165501 (2011).

[88] O. Hellman, I. A. Abrikosov, and S. I. Simak, Lattice Dynamics of Anharmonic Solids from First Principles, Phys. Rev. B 84, 180301 (2011).

[89] I. Errea, M. Calandra, and F. Mauri, Anharmonic Free Energies and Phonon Dispersions from the Stochastic Self-Consistent Harmonic Approximation: Application to Platinum and Palladium Hydrides, Phys. Rev. B 89, 064302 (2014).

[90] D. J. Hooton, LI. A New Treatment of Anharmonicity in Lattice, London, Edinburgh Dublin Philos. Mag. J. Sci. 46, 422 (1955).

[91] T. R. Koehler, Theory of the Self-Consistent Harmonic Approximation with Application to Solid Neon, Phys. Rev. Lett. 17, 89 (1966).

[92] N. R. Werthamer, Self-Consistent Phonon Formulation of Anharmonic Lattice Dynamics, Phys. Rev. B 1, 572 (1970).

[93] M. L. Klein and G. K. Horton, The Rise of Self-Consistent Phonon Theory, J. Low Temp. Phys. 9, 151 (1972).

[94] A. van Roekeghem, J. Carrete, and N. Mingo, Anomalous Thermal Conductivity and Suppression of Negative Thermal Expansion in $\mathrm{ScF}_{3}$, Phys. Rev. B 94, 020303 (2016).

[95] B. Abeles, Lattice Thermal Conductivity of Disordered Semiconductor Alloys at High Temperatures, Phys. Rev. 131, 1906 (1963).

[96] S.-i. Tamura, Isotope Scattering of Dispersive Phonons in Ge, Phys. Rev. B 27, 858 (1983).

[97] S.-i. Tamura, Isotope Scattering of Large-Wave-Vector Phonons in GaAs and InSb: Deformation-Dipole and Overlap-Shell Models, Phys. Rev. B 30, 849 (1984).

[98] J. Klarbring, O. Hellman, I. A. Abrikosov, and S. I. Simak, Anharmonicity and Ultralow Thermal Conductivity in Lead-Free Halide Double Perovskites, Phys. Rev. Lett. 125, 045701 (2020).

[99] X. Yang, T. Feng, J. S. Kang, Y. Hu, J. Li, and X. Ruan, Observation of Strong Higher-Order Lattice Anharmonicity in Raman and Infrared Spectra, Phys. Rev. B 101, 161202 (2020).

[100] N. K. Ravichandran and D. Broido, Non-monotonic Pressure Dependence of the Thermal Conductivity of Boron Arsenide, Nat. Commun. 10, 827 (2019).

[101] Y. Xia, K. Pal, J. He, V. Ozoliņš, and C. Wolverton, Particlelike Phonon Propagation Dominates Ultralow Lattice Thermal Conductivity in Crystalline $\mathrm{Tl}_{3} \mathrm{VSe}_{4}$, Phys. Rev. Lett. 124, 065901 (2020).

[102] Z. Tong, X. Yang, T. Feng, H. Bao, and X. Ruan, FirstPrinciples Predictions of Temperature-Dependent Infrared Dielectric Function of Polar Materials by Including Four-Phonon Scattering and Phonon Frequency Shift, Phys. Rev. B 101, 125416 (2020).

[103] X. Gu, S. Li, and H. Bao, Thermal Conductivity of Silicon at Elevated Temperature: Role of Four-Phonon Scattering and Electronic Heat Conduction, Int. J. Heat Mass Transfer 160, 120165 (2020). 
[104] N. K. Ravichandran and D. Broido, Phonon-Phonon Interactions in Strongly Bonded Solids: Selection Rules and Higher-Order Processes, Phys. Rev. X 10, 021063 (2020).

[105] G. P. Srivastava, Phonon Conductivity Due to Nondiagonal Energy-Flux Operator, J. Phys. (Paris), Colloq. 42, C6 (1981), https://hal.archives-ouvertes.fr/jpa-00221609/document.

[106] B. S. Semwal and P. K. Sharma, Thermal Conductivity of an Anharmonic Crystal, Phys. Rev. B 5, 3909 (1972).

[107] D. C. Knauss and R.S. Wilson, Theory of Thermal Conductivity of Anharmonic Crystals: Nondiagonal Peierls Contribution, Phys. Rev. B 10, 4383 (1974).

[108] G. P. Srivastava and M. Prasad, Diagonal and Nondiagonal Peierls Contribution to the Thermal Conductivity of Anharmonic Crystals, Phys. Rev. B 23, 4273 (1981).

[109] P. B. Allen and J. L. Feldman, Thermal Conductivity of Disordered Harmonic Solids, Phys. Rev. B 48, 12581 (1993).

[110] L. Isaeva, G. Barbalinardo, D. Donadio, and S. Baroni, Modeling Heat Transport in Crystals and Glasses from a Unified Lattice-Dynamical Approach, Nat. Commun. 10, 3853 (2019).

[111] A. Auerbach and P. B. Allen, Universal High-Temperature Saturation in Phonon and Electron Transport, Phys. Rev. B 29, 2884 (1984).

[112] M. Omini and A. Sparavigna, An Iterative Approach to the Phonon Boltzmann Equation in the Theory of Thermal Conductivity, Physica B (Amsterdam) 212, 101 (1995).

[113] M. Omini and A. Sparavigna, Beyond the Isotropic-Model Approximation in the Theory of Thermal Conductivity, Phys. Rev. B 53, 9064 (1996).

[114] W. Li, J. Carrete, N. A. Katcho, and N. Mingo, ShengBTE: A Solver of the Boltzmann Transport Equation for Phonons, Comput. Phys. Commun. 185, 1747 (2014).

[115] Y. Xia, J. M. Hodges, M. G. Kanatzidis, and M. K. Y. Chan, Lattice Thermal Transport in Group II-Alloyed PbTe, Appl. Phys. Lett. 112, 181906 (2018).

[116] G. Kresse and J. Hafner, Ab Initio Molecular Dynamics for Liquid Metals, Phys. Rev. B 47, 558 (1993).

[117] G. Kresse and J. Hafner, Ab Initio Molecular-Dynamics Simulation of the Liquid-Metal-Amorphous-Semiconductor Transition in Germanium, Phys. Rev. B 49, 14251 (1994).

[118] G. Kresse and J. Furthmüller, Comput. Mater. Sci. 6, 15 (1996).

[119] G. Kresse and J. Furthmüller, Efficient Iterative Schemes for Ab Initio Total-Energy Calculations Using a PlaneWave Basis Set, Phys. Rev. B 54, 11169 (1996).

[120] P. E. Blöchl, Projector Augmented-Wave Method, Phys. Rev. B 50, 17953 (1994).

[121] J. P. Perdew, K. Burke, and M. Ernzerhof, Generalized Gradient Approximation Made Simple, Phys. Rev. Lett. 77, 3865 (1996).

[122] J. P. Perdew, K. Burke, and Y. Wang, Generalized Gradient Approximation for the Exchange-Correlation Hole of a Many-Electron System, Phys. Rev. B 54, 16533 (1996).

[123] Y. Wang, J. J. Wang, W. Y. Wang, Z. G. Mei, S. L. Shang, L. Q. Chen, and Z. K. Liu, A Mixed-Space Approach to First-Principles Calculations of Phonon Frequencies for Polar Materials, J. Phys. Condens. Matter 22, 202201 (2010).
[124] S. Baroni and R. Resta, Ab Initio, Phys. Rev. B 33, 7017 (1986).

[125] M. Gajdoš, K. Hummer, G. Kresse, J. Furthmüller, and F. Bechstedt, Linear Optical Properties in the ProjectorAugmented Wave Methodology, Phys. Rev. B 73, 045112 (2006).

[126] J. E. Saal, S. Kirklin, M. Aykol, B. Meredig, and C. Wolverton, Materials Design and Discovery with High-Throughput Density Functional Theory: The Open Quantum Materials Database (OQMD), JOM 65, 1501 (2013).

[127] S. Kirklin, J. E. Saal, B. Meredig, A. Thompson, J. W. Doak, M. Aykol, S. Rühl, and C. Wolverton, The Open Quantum Materials Database (OQMD): Assessing the Accuracy of DFT Formation Energies, NPJ Comput. Mater. 1, 15010 (2015).

[128] A. Seko, A. Togo, H. Hayashi, K. Tsuda, L. Chaput, and I. Tanaka, Prediction of Low-Thermal-Conductivity Compounds with First-Principles Anharmonic LatticeDynamics Calculations and Bayesian Optimization, Phys. Rev. Lett. 115, 205901 (2015).

[129] Y. Pei, X. Shi, A. LaLonde, H. Wang, L. Chen, and G. J. Snyder, Convergence of Electronic Bands for High Performance Bulk Thermoelectrics, Nature (London) 473, 66 (2011).

[130] M. Markov, X. Hu, H.-C. Liu, N. Liu, S. J. Poon, K. Esfarjani, and M. Zebarjadi, Semi-Metals as Potential Thermoelectric Materials, Sci. Rep. 8, 9876 (2018).

[131] C. W. Li, O. Hellman, J. Ma, A. F. May, H. B. Cao, X. Chen, A. D. Christianson, G. Ehlers, D. J. Singh, B. C. Sales, and O. Delaire, Phonon Self-Energy and Origin of Anomalous Neutron Scattering Spectra in SnTe and PbTe Thermoelectrics, Phys. Rev. Lett. 112, 175501 (2014).

[132] G. A. S. Ribeiro, L. Paulatto, R. Bianco, I. Errea, F. Mauri, and M. Calandra, Strong Anharmonicity in the Phonon Spectra of PbTe and SnTe from First Principles, Phys. Rev. B 97, 014306 (2018).

[133] T. Shiga, J. Shiomi, J. Ma, O. Delaire, T. Radzynski, A. Lusakowski, K. Esfarjani, and G. Chen, Microscopic Mechanism of Low Thermal Conductivity in Lead Telluride, Phys. Rev. B 85, 155203 (2012).

[134] G. I. Csonka, J. P. Perdew, A. Ruzsinszky, P. H. T. Philipsen, S. Lebègue, J. Paier, O. A. Vydrov, and J. G. Ángyán, Assessing the Performance of Recent Density Functionals for Bulk Solids, Phys. Rev. B 79, 155107 (2009).

[135] G. Petretto, S. Dwaraknath, H. P. C. Miranda, D. Winston, M. Giantomassi, M. J. van Setten, X. Gonze, K. A. Persson, G. Hautier, and G.-M. Rignanese, High-Throughput Density-Functional Perturbation Theory Phonons for Inorganic Materials, Sci. Data 5, 180065 (2018).

[136] J. Carrete, W. Li, N. Mingo, S. Wang, and S. Curtarolo, Finding Unprecedentedly Low-Thermal-Conductivity Half-Heusler Semiconductors via High-Throughput Materials Modeling, Phys. Rev. X 4, 011019 (2014).

[137] A. van Roekeghem, J. Carrete, C. Oses, S. Curtarolo, and N. Mingo, High-Throughput Computation of Thermal Conductivity of High-Temperature Solid Phases: The Case of Oxide and Fluoride Perovskites, Phys. Rev. X 6, 041061 (2016). 
[138] T. Zhu, S. Gong, T. Xie, P. Gorai, and J. C. Grossman, Charting Lattice Thermal Conductivity of Inorganic Crystals, arXiv:2006.11712.

[139] L. Lindsay and D. A. Broido, Three-Phonon Phase Space and Lattice Thermal Conductivity in Semiconductors, J. Phys. Condens. Matter 20, 165209 (2008).

[140] See http://phonondb.mtl.kyoto-u.ac.jp.

[141] X. Yang, Y. Zhao, Z. Dai, M. Zulfiqar, J. Zhu, and J. Ni, Thermal Expansion Induced Reduction of Lattice Thermal Conductivity in Light Crystals, Phys. Lett. A 381, 3514 (2017).

[142] J. P. Perdew, A. Ruzsinszky, G. I. Csonka, O. A. Vydrov, G. E. Scuseria, L. A. Constantin, X. Zhou, and K. Burke, Restoring the Density-Gradient Expansion for Exchange in Solids and Surfaces, Phys. Rev. Lett. 100, 136406 (2008).

[143] D. B. Benin, Thermal Conductivity of Ne and $\mathrm{He}^{4}$ Quantum Crystals, Phys. Rev. Lett. 20, 1352 (1968).

[144] H. R. Clyde and M. L. Klein, Anharmonic Effects and the Lattice Dynamics of Insulators, CRC Crit. Rev. Solid State Sci. 2, 181 (1971).

[145] R. Bianco, I. Errea, L. Paulatto, M. Calandra, and . Mauri, Second-Order Structural Phase Transitions, Free Energy Curvature, and Temperature-Dependent Anharmonic Phonons in the Self-Consistent Harmonic Approximation: Theory and Stochastic Implementation, Phys. Rev. B 96, 014111 (2017).

[146] O. Hellman, P. Steneteg, I. A. Abrikosov, and S. I. Simak, Temperature Dependent Effective Potential Method for Accurate Free Energy Calculations of Solids, Phys. Rev. B 87, 104111 (2013).

[147] T. Ouyang and M. Hu, First-Principles Study on Lattice Thermal Conductivity of Thermoelectrics HgTe in Different Phases, J. Appl. Phys. 117, 245101 (2015).

[148] H. Kepa, W. Gebicki, T. Giebultowicz, B. Buras, and K. Clausen, A Neutron Study of Phonon Dispersion Relations in HgTe, Solid State Commun. 34, 211 (1980).

[149] H. Kepa, T. Giebultowicz, B. Buras, B. Lebech, and K. Clausen, A Neutron Scattering Study of Lattice Dynamics of HgTe and HgSe, Phys. Scr. 25, 807 (1982).

[150] X. Yang, Z. Dai, Y. Zhao, and S. Meng, Superhigh Thermoelectric Figure of Merit in Silver Halides $\mathrm{AgCl}$ and $\mathrm{AgBr}$ from First Principles, arXiv:1904.06010.

[151] W. Lv and A. Henry, Non-negligible Contributions to Thermal Conductivity from Localized Modes in Amorphous Silicon Dioxide, Sci. Rep. 6, 35720 (2016).

[152] J. L. Niedziela, D. Bansal, A. F. May, J. Ding, T. LaniganAtkins, G. Ehlers, D. L. Abernathy, A. Said, and O. Delaire, Selective Breakdown of Phonon Quasiparticles across Superionic Transition in $\mathrm{CuCrSe}$, Nat. Phys. 15, 73 (2019).

[153] H. R. Clyde and M. L. Klein, Anharmonic Effects and the Lattice Dynamics of Insulators, CRC Crit. Rev. Solid State Sci. 2, 181 (1971).

[154] J. Behler and M. Parrinello, Generalized Neural-Network Representation of High-Dimensional Potential-Energy Surfaces, Phys. Rev. Lett. 98, 146401 (2007).

[155] A. P. Bartók, M. C. Payne, R. Kondor, and G. Csányi, Gaussian Approximation Potentials: The Accuracy of
Quantum Mechanics, without the Electrons, Phys. Rev. Lett. 104, 136403 (2010).

[156] A. V. Shapeev, Moment Tensor Potentials: A Class of Systematically Improvable Interatomic Potentials, Multiscale Model. Simul. 14, 1153 (2016).

[157] L. Zhang, J. Han, H. Wang, R. Car, and E. Weinan, Deep Potential Molecular Dynamics: A Scalable Model with the Accuracy of Quantum Mechanics, Phys. Rev. Lett. 120, 143001 (2018).

[158] M. I. Baskes, Application of the Embedded-Atom Method to Covalent Materials: A Semiempirical Potential for Silicon, Phys. Rev. Lett. 59, 2666 (1987).

[159] M. I. Baskes, J. S. Nelson, and A. F. Wright, Semiempirical Modified Embedded-Atom Potentials for Silicon and Germanium, Phys. Rev. B 40, 6085 (1989).

[160] T. Sun and P. B. Allen, Lattice Thermal Conductivity: Computations and Theory of the High-Temperature Breakdown of the Phonon-Gas Model, Phys. Rev. B 82, 224305 (2010).

[161] P. Haas, F. Tran, and P. Blaha, Calculation of the Lattice Constant of Solids with Semilocal Functionals, Phys. Rev. B 79, 085104 (2009).

[162] Y. Xia, V. Ozoliņ̌s, and C. Wolverton, Microscopic Mechanisms of Glasslike Lattice Thermal Transport in Cubic $\mathrm{Cu}_{12} \mathrm{Sb}_{4} \mathrm{~S}_{13}$ Tetrahedrites, Phys. Rev. Lett. 125, 085901 (2020).

[163] Y. Zhao, C. Lian, S. Zeng, Z. Dai, S. Meng, and J. Ni, Quartic Anharmonicity and Anomalous Thermal Conductivity in Cubic Antiperovskites $A_{3} B \mathrm{O}(A=\mathrm{K}, \mathrm{Rb}$; $B=\mathrm{Br}, \mathrm{Au})$, Phys. Rev. B 101, 184303 (2020).

[164] www.vasp.at.

[165] https://bitbucket.org/sousaw/shengbte.

[166] https://github.com/LLNL/csld.

[167] T. Tadano, Y. Gohda, and S. Tsuneyuki, Anharmonic Force Constants Extracted from First-Principles Molecular Dynamics: Applications to Heat Transfer Simulations, J. Phys. Condens. Matter 26, 225402 (2014).

[168] W. Li, L. Lindsay, D. A. Broido, D. A. Stewart, and N. Mingo, Thermal Conductivity of Bulk and Nanowire $\mathrm{Mg}_{2} \mathrm{Si}_{x} \mathrm{Sn}_{1-x}$ Alloys from First Principles, Phys. Rev. B 86, 174307 (2012).

[169] E. J. Candès and M. B. Wakin, An Introduction to Compressive Sampling, IEEE Signal Process. Mag. 25, 21 (2008).

[170] A. Togo, L. Chaput, and I. Tanaka, Distributions of Phonon Lifetimes in Brillouin Zones, Phys. Rev. B 91, 094306 (2015).

[171] J. He, M. Amsler, Y. Xia, S. S. Naghavi, V. I. Hegde, S. Hao, S. Goedecker, V. Ozoliņš, and C. Wolverton, Ultralow Thermal Conductivity in Full Heusler Semiconductors, Phys. Rev. Lett. 117, 046602 (2016).

[172] T. A. Mellan, A. Aziz, Y. Xia, R. Grau-Crespo, and A. I. Duff, Electron and Phonon Interactions and Transport in the Ultrahigh-Temperature Ceramic ZrC, Phys. Rev. B 99, 094310 (2019).

[173] J. He, Y. Xia, S. S. Naghavi, V. Ozoliņš, and C. Wolverton, Designing Chemical Analogs to PbTe with Intrinsic High Band Degeneracy and Low Lattice Thermal Conductivity, Nat. Commun. 10, 719 (2019). 